\title{
Macrofaunal colonization across the Indian margin oxygen minimum zone
}

\author{
L. A. Levin ${ }^{1}$, A. L. McGregor ${ }^{1}$, G. F. Mendoza ${ }^{1}$, C. Woulds ${ }^{2}{ }^{*}$, P. Cross ${ }^{1}$, U. Witte ${ }^{3}$, A. J. Gooday ${ }^{4}$ G. Cowie $^{2}$, and \\ H. Kitazato ${ }^{5}$ \\ ${ }^{1}$ Center for Marine Biodiversity and Conservation and Integrative Oceanography Division, Scripps Institution of \\ Oceanography, La Jolla, CA 92093-0218, USA \\ ${ }^{2}$ School of Geosciences, The University of Edinburgh, Edinburgh EH9 3JW, UK \\ ${ }^{3}$ Oceanlab, University of Aberdeen, Newburgh, Aberdeenshire AB41 6AA, UK \\ ${ }^{4}$ National Oceanography Centre, Southampton, SO14 3ZH, UK \\ ${ }^{5}$ Japan Agency for Marine-Earth Science and Technology, Natsushima 2-15, Yokosuka, Kanagawa 237-0061, Japan \\ * now at: School of Geography, University of Leeds, Leeds, LS2 9JT, UK
}

Correspondence to: L. A. Levin (llevin@ucsd.edu)

Received: 7 May 2013 - Published in Biogeosciences Discuss.: 13 June 2013

Revised: 26 September 2013 - Accepted: 10 October 2013 - Published: 12 November 2013

\begin{abstract}
There is a growing need to understand the ability of bathyal assemblages to recover from disturbance and oxygen stress, as human activities and expanding oxygen minimum zones increasingly affect deep continental margins. The effects of a pronounced oxygen minimum zone (OMZ) on slope benthic community structure have been studied on every major upwelling margin; however, little is known about the dynamics or resilience of these benthic populations. To examine the influence of oxygen and phytodetritus on shortterm settlement patterns, we conducted colonization experiments at 3 depths on the West Indian continental margin. Four colonization trays were deployed at each depth for 4 days at 542 and $802 \mathrm{~m}$ (transect $1-16^{\circ} 58^{\prime} \mathrm{N}$ ) and for 9 days at 817 and $1147 \mathrm{~m}$ (transect $\left.2-17^{\circ} 31^{\prime} \mathrm{N}\right)$. Oxygen concentrations ranged from $0.9 \mu \mathrm{M}\left(0.02 \mathrm{~mL} \mathrm{~L}^{-1}\right)$ at $542 \mathrm{~m}$ to $22 \mu \mathrm{M}$ $\left(0.5 \mathrm{~mL} \mathrm{~L}^{-1}\right)$ at $1147 \mathrm{~m}$. All trays contained local defaunated sediments; half of the trays at each depth also contained ${ }^{13} \mathrm{C} /{ }^{15} \mathrm{~N}$-labeled phytodetritus mixed into the sediments. Sediment cores were collected between $535 \mathrm{~m}$ and $1140 \mathrm{~m}$ from 2 cross-margin transects for analysis of ambient (source) macrofaunal $(>300 \mu \mathrm{m})$ densities and composition. Ambient macrofaunal densities ranged from 0 ind $\mathrm{m}^{-2}$

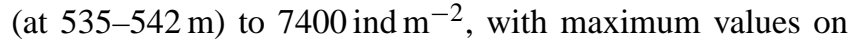
both transects at 700-800 m. Macrofaunal colonizer densities ranged from 0 ind $\mathrm{m}^{-2}$ at $542 \mathrm{~m}$, where oxygen was lowest, to average values of 142 ind $\mathrm{m}^{-2}$ at $800 \mathrm{~m}$, and 3074 ind $\mathrm{m}^{-2}$
\end{abstract}

at $1147 \mathrm{~m}$, where oxygen concentration was highest. These were equal to 4.3 and $151 \%$ of the ambient community at $800 \mathrm{~m}$ and $1147 \mathrm{~m}$, respectively. Community structure of settlers showed no response to the presence of phytodetritus. Increasing depth and oxygen concentration, however, significantly influenced the community composition and abundance of colonizing macrofauna. Polychaetes constituted $92.4 \%$ of the total colonizers, followed by crustaceans $(4.2 \%)$, mollusks $(2.5 \%)$, and echinoderms $(0.8 \%)$. The majority of colonizers were found at $1147 \mathrm{~m} ; 88.5 \%$ of these were Capitella sp., although they were rare in the ambient community. Colonists at 800 and $1147 \mathrm{~m}$ also included ampharetid, spionid, syllid, lumbrinerid, cirratulid, cossurid and sabellid polychaetes. Consumption of ${ }^{13} \mathrm{C} /{ }^{15} \mathrm{~N}$-labeled phytodetritus was observed for macrofaunal foraminifera (too large to be colonizers) at the 542 and $802 / 817 \mathrm{~m}$ sites, and by metazoan macrofauna mainly at the deepest, better oxygenated sites. Calcareous foraminifera (Uvigerina, Hoeglundina sp.), capitellid polychaetes and cumaceans were among the major phytodetritus consumers. These preliminary experiments suggest that bottom-water oxygen concentrations may strongly influence ecosystem services on continental margins, as reflected in rates of colonization by benthos and colonizer processing of carbon following disturbance. They may also provide a window into future patterns of 
settlement on the continental slope as the world's oxygen minimum zones expand.

\section{Introduction}

Oxygen minimum zones (OMZs), areas with $\mathrm{O}_{2}$ concentrations $<0.5 \mathrm{mLL}^{-1}(=22 \mu \mathrm{M})$, blanket a significant fraction of the upper bathyal zone along the eastern Pacific, western Africa and north Indian Ocean continental margins, covering over 1 million square $\mathrm{km}$ of seafloor (Helly and Levin, 2004). There is now recognition that as the planet warms, the oxygen content of the world ocean is declining, a phenomenon termed ocean deoxygenation (Keeling et al., 2010). One consequence is that the world's oxygen minimum zones are expanding (Stramma et al., 2008, 2010) and upper OMZ boundaries are shoaling (Bograd et al., 2008; McClatchie et al., 2010). This means that increasingly larger areas of the continental margin will experience hypoxia or anoxia. Thus, understanding the influence of oxygen on benthic community structure and function will take on added significance. Information about community dynamics and resilience is also of growing importance as margin ecosystems are increasingly subject to direct human disturbance (RamirezLlodra et al., 2011). Physical disturbance from bottom trawling, seabed mining (e.g., of diamonds, phosphates, sulfides, and sand), and chemical disturbance from hydrocarbon spills are current or looming sources of disturbance to bathyal margin ecosystems.

Early studies of OMZs revealed distinct global patterns in which macrofaunal assemblages were characterized by reduced densities at the lowest oxygen levels and density maxima in the lower OMZ transition zone (reviewed in Levin, 2003). Worldwide, OMZs exhibit a high proportion of annelids and low representation of echinoderms (Levin, 2003) with strong diversity shifts linked to oxygen gradients (e.g., Levin et al., 2009). Within the Indian Ocean, these patterns have been reported for the Oman (Levin et al., 2000) and Pakistan (Hughes et al., 2009; Levin et al., 2009) margins, as well as on the W. Indian margin (Ingole et al., 2010; Hunter et al., 2011, 2012) and in the Bay of Bengal (Gooday et al., 2010; A. Raman, unpubl.). In the Arabian Sea investigations, oxygen has been shown to be an important factor limiting the density, body size and taxonomic groups of macrofauna found in OMZs. At OMZ margins in both the Pacific and Indian Oceans, macrofaunal species richness exhibits a positive correlation with bottom-water oxygen concentration, although organic carbon content exerts strong control on evenness and dominance (Levin and Gage, 1998; Levin et al., 2009; A. Raman, personal communication, 2012).

Most studies of OMZ macrobenthos are based on single (or sometimes two) cross-margin transects that provide a static picture of community structure. They do not provide information about whether the structural attributes of
OMZ assemblages described above are generated at settlement, nor information about species interactions and differential mortality that occurs after settlement. Short-term colonization studies can be used to examine settlement potential and preferences as well as successional trends. This approach has been adopted frequently with fouling panels in shallow water (e.g., Pacheco et al., 2010), but is less common in the deep sea.

Deep-water colonization was originally shown to be a relatively slow process compared to shallow depths (Levin and Smith, 1984; Desbruyeres et al., 1985; Grassle and MorsePorteous, 1987; Smith and Hessler, 1987). The stability of different patches in the deep sea was thought to allow for more specialization than in shallow water and to promote succession (Snelgrove et al., 1994). Longer-term (6 months to $>1 \mathrm{yr}$ ) colonization experiments in the deep sea have been performed to determine which taxa are the most efficient colonizers in a given area and to observe the effect of variable food type (Grassle and Morese-Porteous, 1987), organic matter quantity and quality (Snelgrove et al., 1992, 1994, 1996; Menot et al., 2009), hydrodynamics/current and terrain, (Levin and DiBacco, 1995), and sulfide present in sediments (Levin et al., 2006; Menot et al., 2009).

In contrast to the results of early studies, recent work indicates that macrofaunal colonization rates on productive margins or in the presence of enhanced organic matter can be rapid. In situ experiments involving deposition of ${ }^{13} \mathrm{C}$ labeled phytodetritus demonstrated rapid utilization of organic matter on margins (Blair et al., 1996; Levin et al., 1997, 1999; Witte et al., 2003, Aberle and Witte, 2003; Hunter et al., 2012; Pozatto et al., 2013). In the Northwest Atlantic, enriched colonization trays exhibited higher densities than both ambient sediments and unenriched trays (Desbruyères et al., 1980). On the W. African margin, macrofaunal response to the addition of organic matter in colonization trays varied as a function of depth and location, with greater effects of organic enrichment observed at bathyal than abyssal depths (Menot et al., 2009). The type of food present also affected colonization. In the Atlantic, trays containing the diatom Thalassiosira attracted significantly more colonists than those containing the seaweed Sargassum (Snelgrove et al., 1992, 1994). On the Pakistan margin, consumers of phytodetritus varied as a function of oxygen regime (Woulds et al., 2007, 2009), with protists dominating phytodetritus consumption at $\mathrm{O}_{2}$ concentrations below $0.1 \mathrm{~mL} \mathrm{~L}^{-1}(4 \mu \mathrm{M})$ and macrofauna dominating at slightly higher oxygen levels. Given the strong influence of oxygen on macrofaunal community structure and trophic functions, we hypothesized that (1) oxygen availability should influence the rate of colonization and (2) oxygen may alter the influence of phytodetritus on colonization and the processing of phytodetritus by colonizers.

Colonization rates in the deep sea can be enhanced by many factors. These include higher sulfide concentrations at cold seeps (Levin et al., 2006), increased time of experiment deployment, increased rate of sediment transport on 
Table 1. Depth, location, physical characteristics and colonization tray deployment information for experiments conducted on the W. Indian margin. Temperature, salinity and \% POC data are taken from Hunter et al. (2012); these authors made measurements at identical study sites at the same time.

\begin{tabular}{|c|c|c|c|c|c|c|c|c|c|c|c|}
\hline $\begin{array}{l}\text { Depth } \\
\text { (m) }\end{array}$ & Transect & Latitude & Longitude & $\begin{array}{c}\text { Temperature } \\
{ }^{\circ} \mathrm{C}\end{array}$ & Salinity & $\begin{array}{c}\text { Percent } \\
\text { Sand }\end{array}$ & $\begin{array}{l}\text { Percent } \\
\text { Corg }\end{array}$ & $\begin{array}{l}\text { Oxygen } \\
\left(\mathrm{mLL}^{-1}\right)\end{array}$ & $\begin{array}{l}\text { Tray } \\
\text { Numbers }\end{array}$ & $\begin{array}{l}\text { Deployment } \\
\text { Dates (2008) }\end{array}$ & $\begin{array}{l}\text { Exposure } \\
\text { Period (d) }\end{array}$ \\
\hline 540 & 1 & $16^{\circ} 58.82^{\prime} \mathrm{N}$ & $71^{\circ} 55.32^{\prime} \mathrm{E}$ & 12.06 & 35.20 & 44 & 3.24 & $<0.01$ & $\mathrm{~A} 1, \mathrm{~A} 2, \mathrm{~A} 3, \mathrm{~A} 10$ & $7-11 \mathrm{Oct}$ & 4 \\
\hline 802 & 1 & $16^{\circ} 58.73^{\prime} \mathrm{N}$ & $71^{\circ} 52.04^{\prime} \mathrm{E}$ & 9.99 & 35.09 & 9.6 & 5.57 & $0.025-0.055$ & $\mathrm{C} 5, \mathrm{C} 6, \mathrm{C} 7, \mathrm{C}$ & $8-12$ Oct & 4 \\
\hline 817 & 2 & $17^{\circ} 31.49^{\prime} \mathrm{N}$ & $71^{\circ} 10.4^{\prime} \mathrm{E}$ & 9.88 & 35.09 & 26.8 & 4.04 & 0.052 & $11 b, 13 b, 14,15$ & 23 Oct-1 Nov & 9 \\
\hline 1147 & 2 & $17^{\circ} 31.45^{\prime} \mathrm{N}$ & $71^{\circ} 5.04^{\prime} \mathrm{E}$ & 7.03 & 34.82 & 15.7 & 4.34 & 0.48 & $5 b, 6 b, 7 b, 8 b$ & 24 Oct -2 Nov & 9 \\
\hline
\end{tabular}

seamounts (Levin and DiBacco, 1995), increased quantity of organic matter (Levin and Smith, 1984; Menot et al., 2009), and preferred food types (Snelgrove et al., 1992). Six-month exposures of defaunated sediments on seamount and slope habitats in the NE Pacific have shown recovery rates of 50 to $75 \%$ of ambient densities, with most individuals belonging to taxa present in ambient cores (Levin et al., 2006; Levin and DiBacco, 1995), whereas sediment trays collected after only 7 weeks yielded densities as low as $6 \%$ of ambient levels in coarse-grained seamount sediments (Levin and DiBacco, 1995). Colonization experiments in the low-oxygen Santa Catalina Basin also yielded assemblages containing a significant proportion of ambient fauna (Levin and Smith, 1984). In contrast, early colonization tray experiments in the Atlantic revealed abundant, opportunistic colonizers that were often rare in ambient sediments (Desbruyères et al., 1980; Grassle and Morse-Porteous, 1987; Snelgrove et al., 1992). These included capitellid, hesionid, dorvilleid, spionid, and sigalionid polychaetes, cumaceans and leptostracans.

This study examines the effects of oxygen and water depth, as well as the presence of phytodetritus on macrobenthos distributions and colonization patterns across the Indian margin OMZ. It is the first to document the initial steps of recolonization at different oxygen levels across an OMZ. Trays were deployed on the sea floor for 4 and 9 days while most other deep-water experiments left trays out for 6 months or longer. In this experiment we tested the null hypotheses for macrofauna that (1) oxygen and depth are not correlated with colonizer abundance, composition, diversity and lifestyle; and that (2) the type and abundance of animals colonizing the trays is unaffected by the presence of phytodetritus. Our alternative hypotheses were that the abundance of organisms would increase with oxygen concentration and depth, there would be a significant difference in community composition between different depths and oxygen concentrations, and that trays containing phytodetritus would support more colonizers. Both macrofaunal and foraminiferal uptake of phytodetritus was examined through the introduction of ${ }^{13} \mathrm{C}$-labeled diatoms in a subset of colonization trays deployed across an oxygen gradient. Depending on the nature of seafloor disturbance, early colonists (in nature) may experience locally enhanced availability of phytodetritus (e.g., accumulating in pits) or a decrease due to removal of surficial sediments or diminished surface production. These experiments explore both the influence of phytodetritus on the colonization process and the extent to which a "disturbance"-adapted fauna may function to process carbon. We also examined the density and composition of macrofauna in ambient sediments along two cross-margin transects to better understand the source assemblages available to colonize the trays.

\section{Methods}

\subsection{Field conditions}

Studies were conducted on the Indian margin in October and November 2008 aboard the RV Yokosuka with the submersible Shinkai 6500. Site characteristics are presented in Table 1 and reviewed in Hunter et al. $(2011 ; 2012)$. Salinity and temperature data were obtained with a Seabird SBE 19 CTD. Temperature near the seabed ranged from 7.03 to $12.06^{\circ} \mathrm{C}$ and salinity near the seabed ranged from 34.82 to 35.20. Oxygen concentrations were measured with an Aanderaa Optode 3830 mounted on the Shinkai 6500; calibration procedures are described in Hunter et al. (2011). Oxygen ranged from $0.35 \mu \mathrm{M}$ at $530 \mathrm{~m}$ to $21.1 \mu \mathrm{M}$ at $1147 \mathrm{~m}$ (see Table 1). Sediments were coarsest ( $44.5 \%$ sand) with lowest organic $\mathrm{C}$ content $(3.24 \%$ ) at the $542 \mathrm{~m}$ (transect 1) site and finest (9.6 \% sand) with highest organic C content (5.71\%) at $802 \mathrm{~m}$ on transect 1 . The 817 and $1147 \mathrm{~m}$ sites on transect 2 had sand content (26.8 and $15.7 \%$ ) and organic $\mathrm{C}$ content (4.04-4.35) that were intermediate between those of the transect 1 sites.

In the study region megafauna and macrofauna are rare at $500-600 \mathrm{~m}$ where oxygen concentration is lowest. Megafauna (animals visible in video imagery) exhibit maximum densities at $800 \mathrm{~m}$ and decline at greater depths; however, mega-infauna and lebensspuren are common only below $1100 \mathrm{~m}$ on both transects (Hunter et al., 2011, 2012).

\subsection{Colonization experiments}

Colonization experiments were placed at locations along two cross-margin transects at slightly different latitudes (Fig. 1). On transect 1 , four colonization trays were deployed for 4 days, from October 7-11 at a depth of $542 \mathrm{~m}$ and from Oct. $8-12$ at $802 \mathrm{~m}$. On transect 2 , four trays were deployed from 23 October to 1 November at a depth of $1147 \mathrm{~m}$ and from 
Table 2. Date, dive number, depth and location of background sediment cores collected for macrofaunal analysis. Each row reflects a single core.

\begin{tabular}{|c|c|c|c|c|}
\hline Date & Dive No. & Depth (m) & Latitude & Longitude \\
\hline TRANSECT 1 & & & ${ }^{\circ} \mathrm{N}$ & ${ }^{\circ} \mathrm{E}$ \\
\hline 6 Oct 2008 & 1103 & 535 & $16^{\circ} 58.77^{\prime}$ & $71^{\circ} 55.41^{\prime}$ \\
\hline 7 Oct 2008 & 1104 & 542 & $16^{\circ} 58.82^{\prime}$ & $71^{\circ} 55.32^{\prime}$ \\
\hline 7 Oct 2008 & 1104 & 542 & $16^{\circ} 58.82^{\prime}$ & $71^{\circ} 55.32^{\prime}$ \\
\hline 4 Oct 2008 & 1102 & 602 & $16^{\circ} 58.82^{\prime}$ & $71^{\circ} 54.63^{\prime}$ \\
\hline 4 Oct 2008 & 1102 & 602 & $16^{\circ} 58.82^{\prime}$ & $71^{\circ} 54.63^{\prime}$ \\
\hline 3 Oct 2008 & 1101 & 710 & $16^{\circ} 58.67^{\prime}$ & $71^{\circ} 53.24^{\prime}$ \\
\hline 3 Oct 2008 & 1101 & 712 & $16^{\circ} 58.67^{\prime}$ & $71^{\circ} 53.24^{\prime}$ \\
\hline 3 Oct 2008 & 1101 & 800 & $16^{\circ} 58.60^{\prime}$ & $71^{\circ} 52.06^{\prime}$ \\
\hline 8 Oct 2008 & 1105 & 802 & $16^{\circ} 58.71^{\prime}$ & $71^{\circ} 52.04^{\prime}$ \\
\hline 2 Oct 2008 & 1100 & 835 & $16^{\circ} 58.90^{\prime}$ & $71^{\circ} 51.96^{\prime}$ \\
\hline 2 Oct 2008 & 1100 & 835 & $16^{\circ} 58.90^{\prime}$ & $71^{\circ} 51.96^{\prime}$ \\
\hline 2 Oct 2008 & 1100 & 900 & $16^{\circ} 58.98^{\prime}$ & $71^{\circ} 51.13^{\prime}$ \\
\hline 1 Oct 2008 & 1099 & 1000 & $16^{\circ} 58.97^{\prime}$ & $71^{\circ} 49.93^{\prime}$ \\
\hline 1 Oct 2008 & 1099 & 1100 & $16^{\circ} 58.89^{\prime}$ & $71^{\circ} 48.58^{\prime}$ \\
\hline TRANSECT 2 & & & ${ }^{\circ} \mathrm{N}$ & ${ }^{\circ} \mathrm{E}$ \\
\hline 8 Nov 2008 & 1123 & 575 & $17^{\circ} 33.33^{\prime}$ & $71^{\circ} 11.55^{\prime}$ \\
\hline 8 Nov 2008 & 1123 & 575 & $17^{\circ} 33.33^{\prime}$ & $71^{\circ} 11.55^{\prime}$ \\
\hline 8 Nov 2008 & 1123 & 650 & N/A & N/A \\
\hline 28 Oct 2008 & 1117 & 693 & $17^{\circ} 32.19^{\prime}$ & $71^{\circ} 10.61^{\prime}$ \\
\hline 28 Oct 2008 & 1117 & 746 & $17^{\circ} 31.99^{\prime}$ & $71^{\circ} 10.52^{\prime}$ \\
\hline 28 Oct 2008 & 1117 & 746 & $17^{\circ} 31.99^{\prime}$ & $71^{\circ} 10.52^{\prime}$ \\
\hline 26 Oct 2008 & 1115 & 813 & $17^{\circ} 31.49^{\prime}$ & $71^{\circ} 10.18^{\prime}$ \\
\hline 23 Oct 2008 & 1112 & 816 & $17^{\circ} 31.50^{\prime}$ & $71^{\circ} 10.45^{\prime}$ \\
\hline 16 Oct 2008 & 1110 & 930 & $17^{\circ} 32.58^{\prime}$ & $71^{\circ} 6.30^{\prime}$ \\
\hline 16 Oct 2008 & 1110 & 930 & $17^{\circ} 32.58^{\prime}$ & $71^{\circ} 6.30^{\prime}$ \\
\hline 27 Oct 2008 & 1116 & 1000 & $17^{\circ} 31.82^{\prime}$ & $71^{\circ} 6.01^{\prime}$ \\
\hline 27 Oct 2008 & 1116 & 1000 & $17^{\circ} 31.82^{\prime}$ & $71^{\circ} 6.01^{\prime}$ \\
\hline 27 Oct 2008 & 1116 & 1050 & $17^{\circ} 31.78^{\prime}$ & $71^{\circ} 5.62^{\prime}$ \\
\hline 22 Oct 2008 & 1111 & 1147 & $17^{\circ} 31.46^{\prime}$ & $71^{\circ} 5.05^{\prime}$ \\
\hline 22 Oct 2008 & 1111 & 1147 & $17^{\circ} 31.46^{\prime}$ & $71^{\circ} 5.05^{\prime}$ \\
\hline
\end{tabular}

24 October to 2 November at $817 \mathrm{~m}$ (Table 1). The colonization trays consisted of an $11.1 \mathrm{~cm}$ diameter central cup $(9 \mathrm{~cm}$ deep) lined with $20 \mu \mathrm{m}$ mesh, surrounded by a flat delrin nylon collar $40 \mathrm{~cm}$ in diameter. The design of the colonization trays is described by Levin and DiBacco (1995) and is identical to those used by Snelgrove et al. (1992, 1994, 1996) in the Atlantic Ocean and Levin et al. $(2006,2013)$ in the Pacific Ocean. The broad collar is designed to reduce turbulent flow over the central cup and prevent scour. Trays were nestled into sediments such that the sediment surface of the cup and the collar were flush with the surrounding sediment. Trays were covered with water-tight lids during deployment and recovery to prevent loss of sediment.

Among the 4 colonization trays deployed at each depth, two each received additions of freeze-dried phytodetritus, made from the diatom Thalassiosira weissflogii labeled with ${ }^{13} \mathrm{C}$ and ${ }^{15} \mathrm{~N}$ (see Hunter et al., 2012 for preparation details); the other two had no algae added. In preparing the colonization trays, sediments were collected from the study sites by scoop and stored on board ship at -80 to $-20^{\circ} \mathrm{C}$ for $1-3$ days. They were then thawed on deck at $30^{\circ} \mathrm{C}$ and sonicated for $5 \mathrm{~min}$ to destroy foraminifera and metazoans. Algae were mixed in $50 \mathrm{cc}$ tubes with $\sim 50 \mathrm{cc}$ of mud and spread on the tray surface immediately before deployment in a $0.25 \mathrm{~mm}$ thick surface layer. The trays contained additions equivalent to $\sim 500 \mathrm{mg} \mathrm{C} \mathrm{m}^{-2}$, roughly the $\mathrm{C}$ input for a single year at the $500 \mathrm{~m}$ site. These doses were similar to those used in experiments on the Pakistan margin (e.g., Woulds et al., 2007) and reflect the pulsed nature of natural organic matter delivery in the Arabian Sea. Sediment-filled trays were recovered and the cup contents were sectioned vertically at $0-1,1-2$, $2-3$, and $3-5 \mathrm{~cm}$. All samples were preserved in $8 \%$ buffered formalin.

\subsection{Ambient faunal collection}

Ambient fauna were collected in October and November 2008 using $8.3 \mathrm{~cm}$ diameter tube cores deployed from the 


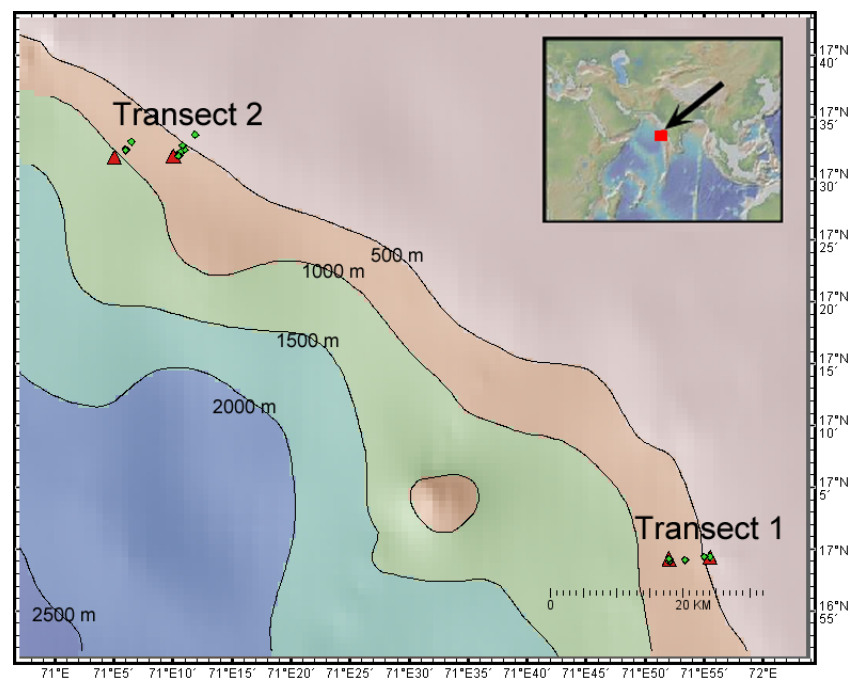

Fig. 1. Location of transects 1 and 2 with colonization trays (red triangles) and ambient cores (green circles) on the W. Indian margin.

Shinkai 6500 (Table 2). Samples from transect $1\left(16^{\circ} 58^{\prime} \mathrm{N}\right.$, $\left.71^{\circ} 55^{\prime} \mathrm{E}\right)$ were collected roughly $100 \mathrm{~km}$ southeast of transect $2\left(17^{\circ} 31^{\prime} \mathrm{N}, 71^{\circ} 10^{\prime} \mathrm{E}\right)$ (Fig. 1). Pairs of cores were taken by the Shinkai 6500 at a range of depths between 500 and $1150 \mathrm{~m}$ (Table 2). Tube core sediments were sectioned vertically on board ship at $0-1,1-2,2-3,3-5$, and $5-10 \mathrm{~cm}$ intervals and fauna were preserved in $8 \%$ buffered formalin. All fractions were sieved in the laboratory through a $300 \mu \mathrm{m}$ mesh and macrofauna were removed from retained sediments under a dissecting microscope. Animals were counted and identified to the lowest taxon possible.

\subsection{Shipboard and laboratory analyses}

The upper fractions were sieved in the laboratory through a $300 \mu \mathrm{m}$ mesh to separate out macrofauna and through a $45 \mu \mathrm{m}$ mesh to retain smaller organisms for later meiofaunal studies. Macrofauna were sorted under a binocular microscope, counted, and identified to the lowest taxon possible. Colonization tray samples were sorted to consecutive $1 \mathrm{~cm}$ depth fractions until no more animals were present. All trays from transect 1 and the $800 \mathrm{~m}$ trays from transect 2 were thus sorted to only $2 \mathrm{~cm}$, as no animals were present below $1 \mathrm{~cm}$. The $1147 \mathrm{~m}$ trays from transect 2 were sorted to $3 \mathrm{~cm}$ ( 2 trays) and $5 \mathrm{~cm}$ ( 2 trays.) Stable isotope analysis was performed on the organisms found in trays containing labeled algae to determine which taxa consumed phytodetritus.

\subsection{Statistical testing}

Multivariate community analysis was performed using Primer software V. 6. Bray-Curtis similarity indices were used to create similarity matrices from untransformed family abundance values. Differences in community composition

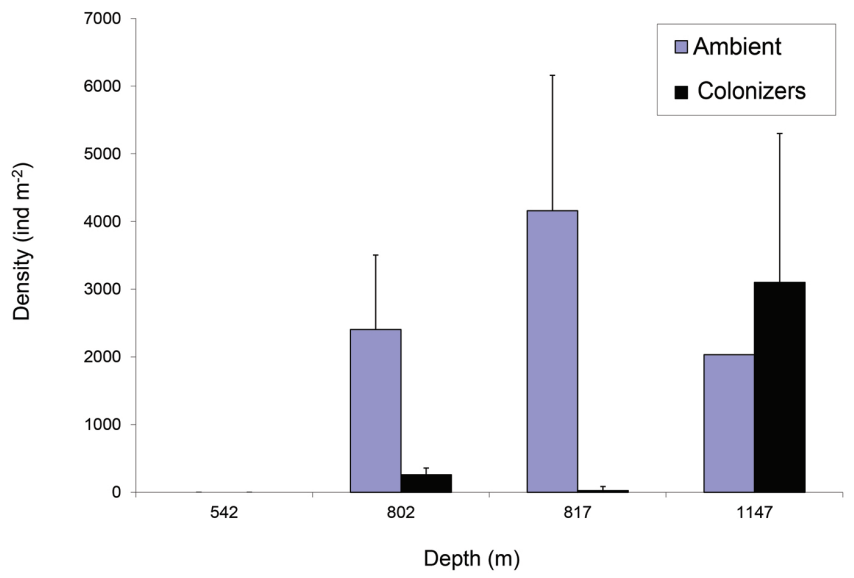

Fig. 2. Comparison of metazoan macrofaunal ambient and colonizer average density ( $\pm 1 \mathrm{SD}$ ) on the W. Indian margin. See Table 1 for tray deployment periods and locations.

are presented in MDS plots. ANOSIM and SIMPER were used to measure sources and statistical significance of the differences in community composition between colonizer and ambient communities, colonizers at higher vs. lower oxygen concentration, and colonizers of trays with and without labeled phytodetritus. The differences between the proportion of polychaetes and densities between colonizer and ambient fauna at a given depth were measured by performing one tailed $t$ tests using JMP software.

\subsection{Stable isotope analyses}

Macrofauna from ambient sediments were sorted, identified and frozen on board ship, prior to stable isotope analyses. Foraminifera from the $0-1 \mathrm{~cm}$ fraction in 542 and $802 \mathrm{~m}$ trays on transect 1 were also sorted and frozen on board. Isotopic analyses of tray colonizers were performed after animals had been preserved in $8 \%$ buffered formalin, as it was not possible to process them all at sea. Formalin introduces minimal alteration of isotope signatures, with shifts in $\delta^{13} \mathrm{C}$ of $-0.5 \%$, and $\delta^{15} \mathrm{~N}$ of $+0.14 \%$ (Levin et al., 2006). In contrast, uptake of isotopically labeled algae (with $\delta^{13} \mathrm{C}$ and $\delta^{15} \mathrm{~N}$ values greater than $50000 \%$ ) creates a signature in consumers that is many orders of magnitude larger than the small formalin shift. Infauna were handled with methanol-dipped forceps, rinsed in Milli-Q water, and placed in preweighed tin boats. Multiple individuals were combined for small taxa. Specimens were dried, weighed on a Sartorius CP2250 balance and then, prior to $\delta^{13} \mathrm{C}$ and $\delta^{15} \mathrm{~N}$ analysis, they were acidified with one drop of $1 \% \mathrm{PtCl}_{2}$ to remove inorganic carbon. Samples were run on a Costech elemental analyzer interfaced with a continuous-flow Micromass Isoprime isotope ratio mass spectrometer at Washington State University (by R. Lee). Isotope ratios are expressed as $\delta^{13} \mathrm{C}$ or $\delta^{15} \mathrm{~N}$ in units of per mil (\%o). Standards were PeeDee Belemnite for $\delta^{13} \mathrm{C}$ and nitrogen gas for $\delta^{15} \mathrm{~N}$ (atmospheric). 
Table 3a. Background macrofaunal densities (no. per $8.3 \mathrm{~cm}$ diameter core), transect 1 (see Table 1 for locations).

\begin{tabular}{|c|c|c|c|c|c|c|c|c|c|c|c|c|c|c|}
\hline TRANSECT 1 & $\begin{array}{l}6 \text { Oct } \\
2008\end{array}$ & $\begin{array}{l}7 \text { Oct } \\
2008\end{array}$ & $\begin{array}{l}7 \text { Oct } \\
2008\end{array}$ & $\begin{array}{l}4 \text { Oct } \\
2008\end{array}$ & $\begin{array}{l}4 \text { Oct } \\
2008\end{array}$ & $\begin{array}{l}3 \text { Oct } \\
2008\end{array}$ & $\begin{array}{l}3 \text { Oct } \\
2008\end{array}$ & $\begin{array}{l}3 \text { Oct } \\
2008\end{array}$ & $\begin{array}{l}8 \text { Oct } \\
2008\end{array}$ & $\begin{array}{l}2 \text { Oct } \\
2008\end{array}$ & $\begin{array}{l}2 \text { Oct } \\
2008\end{array}$ & $\begin{array}{l}2 \text { Oct } \\
2008\end{array}$ & $\begin{array}{l}1 \text { Oct } \\
2008\end{array}$ & $\begin{array}{l}1 \text { Oct } \\
2008\end{array}$ \\
\hline Depth (m) & 535 & 542 & 542 & 602 & 602 & 710 & 712 & 800 & 802 & 835 & 835 & 900 & 1000 & 1100 \\
\hline \multicolumn{15}{|l|}{ Annelida } \\
\hline Acrocirridae & 0 & 0 & 0 & 0 & 0 & 0 & 0 & 0 & 0 & 0 & 0 & 0 & 0 & 0 \\
\hline Ampharetidae & 0 & 0 & 0 & 0 & 0 & 6 & 4 & 1 & 0 & 0 & 1 & 0 & 0 & 0 \\
\hline Amphinomidae & 0 & 0 & 0 & 0 & 0 & 0 & 0 & 0 & 0 & 0 & 0 & 0 & 0 & 0 \\
\hline Capitellidae & 0 & 0 & 0 & 0 & 0 & 0 & 0 & 0 & 1 & 0 & 0 & 0 & 0 & 0 \\
\hline Cirratullidae & 0 & 0 & 0 & 0 & 0 & 0 & 5 & 2 & 9 & 2 & 3 & 1 & 0 & 0 \\
\hline Cossuridae & 0 & 0 & 0 & 0 & 0 & 2 & 8 & 1 & 2 & 0 & 1 & 2 & 0 & 0 \\
\hline Fauveliopsidae & 0 & 0 & 0 & 0 & 0 & 0 & 0 & 0 & 0 & 0 & 0 & 0 & 0 & 0 \\
\hline Hesionidae & 0 & 0 & 0 & 0 & 0 & 0 & 0 & 0 & 0 & 0 & 0 & 0 & 1 & 0 \\
\hline Lumbrineridae & 0 & 0 & 0 & 0 & 0 & 0 & 0 & 1 & 1 & 0 & 0 & 0 & 0 & 0 \\
\hline Maldanidae & 0 & 0 & 0 & 0 & 0 & 0 & 0 & 0 & 0 & 0 & 0 & 0 & 0 & 0 \\
\hline Nephtyidae & 0 & 0 & 0 & 0 & 0 & 0 & 2 & 0 & 0 & 0 & 0 & 0 & 1 & 0 \\
\hline Nereididae & 0 & 0 & 0 & 0 & 0 & 0 & 0 & 0 & 0 & 0 & 0 & 0 & 1 & 0 \\
\hline Paraonidae & 0 & 0 & 0 & 0 & 0 & 0 & 0 & 2 & 4 & 4 & 5 & 0 & 0 & 0 \\
\hline Pectinaridae & 0 & 0 & 0 & 0 & 0 & 0 & 0 & 0 & 0 & 0 & 0 & 0 & 0 & 0 \\
\hline Phyllodocidae & 0 & 0 & 0 & 0 & 0 & 0 & 0 & 0 & 0 & 0 & 0 & 0 & 0 & 0 \\
\hline Polynoidae & 0 & 0 & 0 & 0 & 0 & 0 & 0 & 0 & 0 & 0 & 0 & 1 & 0 & 1 \\
\hline Sabellidae & 0 & 0 & 0 & 0 & 0 & 2 & 1 & 1 & 0 & 0 & 0 & 0 & 0 & 0 \\
\hline Scalibregmatidae & 0 & 0 & 0 & 0 & 0 & 0 & 0 & 0 & 0 & 0 & 0 & 0 & 0 & 0 \\
\hline Sigalionidae & 0 & 0 & 0 & 0 & 0 & 0 & 0 & 0 & 0 & 0 & 0 & 0 & 0 & 0 \\
\hline Sphaerodoridae & 0 & 0 & 0 & 0 & 0 & 0 & 0 & 0 & 0 & 0 & 0 & 3 & 0 & 0 \\
\hline Spionidae & 0 & 0 & 0 & 12 & 0 & 3 & 0 & 2 & 1 & 0 & 1 & 0 & 0 & 0 \\
\hline Sternaspidae & 0 & 0 & 0 & 0 & 0 & 0 & 0 & 0 & 0 & 0 & 1 & 0 & 0 & 0 \\
\hline Syllidae & 0 & 0 & 0 & 1 & 1 & 0 & 0 & 0 & 0 & 0 & 0 & 0 & 1 & 0 \\
\hline Trichobranchidae & 0 & 0 & 0 & 0 & 0 & 0 & 0 & 0 & 0 & 0 & 0 & 0 & 0 & 0 \\
\hline \multicolumn{15}{|l|}{ Crustacea } \\
\hline Cumacea & 0 & 0 & 0 & 0 & 0 & 0 & 0 & 0 & 0 & 0 & 0 & 0 & 0 & 0 \\
\hline Isopoda & 0 & 0 & 0 & 0 & 0 & 0 & 0 & 0 & 0 & 0 & 0 & 0 & 0 & 0 \\
\hline Amphipoda & 0 & 0 & 0 & 0 & 0 & 3 & 1 & 0 & 0 & 3 & 0 & 0 & 0 & 0 \\
\hline Tanaidacia & 0 & 0 & 0 & 0 & 0 & 0 & 0 & 0 & 0 & 0 & 0 & 0 & 0 & 12 \\
\hline \multicolumn{15}{|l|}{ Arachnida } \\
\hline Mite & 0 & 0 & 0 & 0 & 0 & 0 & 0 & 0 & 0 & 0 & 0 & 0 & 0 & 0 \\
\hline \multicolumn{15}{|l|}{ Mollusca } \\
\hline Aplacophora & 0 & 0 & 0 & 0 & 0 & 2 & 0 & 0 & 0 & 0 & 0 & 0 & 1 & 0 \\
\hline Bivalvia & 0 & 0 & 0 & 0 & 0 & 11 & 5 & 0 & 0 & 0 & 0 & 0 & 0 & 0 \\
\hline Lucinidae & 0 & 0 & 0 & 0 & 0 & 0 & 0 & 0 & 0 & 0 & 0 & 0 & 0 & 0 \\
\hline Pectinidae & 0 & 0 & 0 & 0 & 0 & 0 & 0 & 0 & 0 & 0 & 0 & 0 & 0 & 0 \\
\hline \multicolumn{15}{|l|}{ Echinodermata } \\
\hline Ophiuroidea & 0 & 0 & 0 & 0 & 0 & 1 & 0 & 0 & 0 & 0 & 0 & 0 & 1 & 0 \\
\hline Nemertean & 0 & 0 & 0 & 0 & 0 & 0 & 0 & 0 & 0 & 0 & 0 & 0 & 0 & 0 \\
\hline Unknown Metazoan & 0 & 0 & 0 & 0 & 0 & 0 & 0 & 0 & 3 & 0 & 0 & 0 & 0 & 1 \\
\hline
\end{tabular}

\section{Results}

\subsection{Colonizer and ambient densities}

Rates of macrofaunal colonization were greater where oxygen was higher on both transects. On transect 1 after 4 days exposure, no metazoan macrofaunal individuals were found in trays at $542 \mathrm{~m}$ depth; this is consistent with the absence of metazoan macrofauna at a comparable depth in ambient sediments (Figs. 2, 3). Average colonizer density was 258.3 ind m $^{-2}$ at $802 \mathrm{~m}$ on transect 1 after 4 days and an order of magnitude lower $\left(25.8 \mathrm{ind}^{-2}\right)$ at $817 \mathrm{~m}$ on tran- sect 2 after 9 days (Table 4). The colonizer densities were $10.7 \%$ and $0.62 \%$ of ambient densities for transects 1 and 2 , respectively (Fig. 2), but significantly lower than ambient densities only on transect $1(P=0.012)$ and not on transect $2(P=0.110)$. Colonizer densities were considerably higher $\left(3074.3\right.$ ind $^{-2}$ ) at $1147 \mathrm{~m}$ on transect 2 ; they were approximately $151 \%$ of ambient density but not significantly different ( $P=0.39$ ) (Fig. 2). Overall, colonization densities were over 20 times higher at $1147 \mathrm{~m}$ than $802-$ $817 \mathrm{~m}\left(t_{10}=4.11 P=0.002\right)$. Metazoan macrofaunal densities in ambient sediments were 0 ind $^{-2}$ from $500-550$ $\mathrm{m}$, increased from 575 to $700 \mathrm{~m}$ to $>4000$ ind $^{-2}$, then 
Table 3b. Background macrofaunal densities (no. per $8.3 \mathrm{~cm}$ diameter core), transect 2 (see Table 1 for locations).

\begin{tabular}{|c|c|c|c|c|c|c|c|c|c|c|c|c|c|c|c|}
\hline TRANSECT 2 & $\begin{array}{l}8 \mathrm{Nov} \\
2008\end{array}$ & $\begin{array}{l}8 \mathrm{Nov} \\
2008\end{array}$ & $\begin{array}{c}8 \mathrm{Nov} \\
2008\end{array}$ & $\begin{array}{c}28 \text { Oct } \\
2008\end{array}$ & $\begin{array}{c}28 \mathrm{Oct} \\
2008\end{array}$ & $\begin{array}{c}28 \text { Oct } \\
2008\end{array}$ & $\begin{array}{c}26 \mathrm{Oct} \\
2008\end{array}$ & $\begin{array}{c}23 \mathrm{Oct} \\
2008\end{array}$ & $\begin{array}{c}16 \text { Oct } \\
2008\end{array}$ & $\begin{array}{c}16 \text { Oct } \\
2008\end{array}$ & $\begin{array}{c}27 \text { Oct } \\
2008\end{array}$ & $\begin{array}{c}27 \text { Oct } \\
2008\end{array}$ & $\begin{array}{c}27 \text { Oct } \\
2008\end{array}$ & $\begin{array}{c}22 \mathrm{Oct} \\
2008\end{array}$ & $\begin{array}{c}22 \mathrm{Oct} \\
2008\end{array}$ \\
\hline Depth (m) & 575 & 575 & 650 & 693 & 746 & 746 & 800 & 816 & 930 & 930 & 1000 & 1000 & 1050 & 1147 & 1147 \\
\hline \multicolumn{16}{|l|}{ Annelida } \\
\hline Acrocirridae & 0 & 0 & 0 & 1 & 0 & 0 & 0 & 0 & 0 & 0 & 0 & 0 & 0 & 0 & 0 \\
\hline Ampharetidae & 0 & 0 & 12 & 8 & 0 & 1 & 2 & 2 & 0 & 0 & 0 & 0 & 0 & 0 & 0 \\
\hline Amphinomidae & 0 & 1 & 1 & 0 & 0 & 0 & 0 & 0 & 0 & 0 & 0 & 0 & 0 & 0 & 0 \\
\hline Capitellidae & 0 & 0 & 0 & 0 & 0 & 0 & 0 & 0 & 1 & 1 & 0 & 0 & 0 & 0 & 0 \\
\hline Cirratullidae & 0 & 0 & 1 & 1 & 2 & 4 & 5 & 3 & 0 & 0 & 0 & 0 & 0 & 0 & 0 \\
\hline Cossuridae & 0 & 0 & 0 & 0 & 0 & 0 & 2 & 0 & 0 & 0 & 0 & 0 & 0 & 0 & 1 \\
\hline Fauveliopsidae & 0 & 0 & 0 & 3 & 0 & 0 & 2 & 0 & 1 & 0 & 0 & 0 & 0 & 0 & 0 \\
\hline Hesionidae & 0 & 0 & 0 & 2 & 0 & 0 & 0 & 0 & 0 & 0 & 0 & 0 & 0 & 0 & 0 \\
\hline Lumbrineridae & 0 & 0 & 0 & 0 & 0 & 0 & 0 & 0 & 0 & 0 & 1 & 0 & 0 & 0 & 0 \\
\hline Maldanidae & 0 & 0 & 0 & 0 & 0 & 0 & 1 & 0 & 2 & 0 & 0 & 0 & 0 & 0 & 0 \\
\hline Nephtyidae & 0 & 0 & 0 & 0 & 0 & 0 & 0 & 0 & 0 & 0 & 0 & 0 & 0 & 1 & 0 \\
\hline Nereididae & 0 & 0 & 0 & 0 & 0 & 0 & 0 & 1 & 0 & 0 & 0 & 0 & 0 & 0 & 0 \\
\hline Paraonidae & 0 & 0 & 0 & 0 & 0 & 0 & 2 & 3 & 0 & 0 & 0 & 1 & 0 & 0 & 3 \\
\hline Pectinaridae & 0 & 0 & 0 & 0 & 2 & 0 & 0 & 0 & 0 & 0 & 0 & 0 & 0 & 0 & 0 \\
\hline Phyllodocidae & 0 & 0 & 0 & 0 & 0 & 0 & 0 & 0 & 0 & 1 & 0 & 0 & 0 & 0 & 0 \\
\hline Polynoidae & 0 & 0 & 0 & 1 & 0 & 0 & 0 & 0 & 0 & 0 & 0 & 0 & 0 & 0 & 0 \\
\hline Sabellidae & 0 & 0 & 2 & 2 & 1 & 1 & 1 & 0 & 1 & 0 & 1 & 0 & 0 & 0 & 1 \\
\hline Scalibregmatidae & 0 & 0 & 0 & 1 & 0 & 0 & 0 & 0 & 0 & 0 & 0 & 0 & 0 & 0 & 0 \\
\hline Sigalionidae & 0 & 0 & 0 & 0 & 0 & 0 & 0 & 0 & 0 & 0 & 0 & 0 & 0 & 0 & 1 \\
\hline Sphaerodoridae & 0 & 0 & 0 & 0 & 0 & 0 & 0 & 0 & 0 & 0 & 0 & 0 & 0 & 0 & 0 \\
\hline Spionidae & 5 & 3 & 4 & 0 & 0 & 0 & 0 & 0 & 0 & 1 & 2 & 1 & 1 & 0 & 1 \\
\hline Sternaspidae & 0 & 0 & 0 & 0 & 0 & 0 & 0 & 0 & 0 & 0 & 0 & 0 & 0 & 0 & 0 \\
\hline Syllidae & 1 & 2 & 0 & 6 & 0 & 0 & 0 & 0 & 1 & 0 & 0 & 2 & 0 & 4 & 0 \\
\hline Trichobranchidae & 0 & 0 & 0 & 2 & 0 & 0 & 0 & 0 & 0 & 0 & 0 & 1 & 0 & 0 & 0 \\
\hline \multicolumn{16}{|l|}{ Crustacea } \\
\hline Cumacean & 0 & 0 & 0 & 0 & 0 & 0 & 0 & 0 & 1 & 0 & 0 & 0 & 0 & 0 & 0 \\
\hline Isopod & 0 & 0 & 0 & 0 & 0 & 0 & 0 & 0 & 0 & 0 & 0 & 0 & 0 & 1 & 0 \\
\hline Amphipoda & 0 & 0 & 0 & 1 & 0 & 0 & 2 & 2 & 0 & 0 & 0 & 0 & 1 & 2 & 0 \\
\hline Tanaid & 0 & 0 & 0 & 0 & 0 & 0 & 0 & 0 & 1 & 0 & 0 & 0 & 0 & 2 & 0 \\
\hline Ampeliscid & 0 & 0 & 0 & 2 & 2 & 1 & 0 & 1 & 0 & 0 & 0 & 0 & 0 & 0 & 0 \\
\hline Stenothoidae & 0 & 0 & 0 & 0 & 0 & 0 & 1 & 0 & 0 & 0 & 0 & 0 & 0 & 0 & 0 \\
\hline Phoxocephalidae & 0 & 0 & 0 & 0 & 0 & 0 & 0 & 0 & 0 & 0 & 0 & 0 & 0 & 0 & 2 \\
\hline Arachnida & 0 & 0 & 0 & 0 & 0 & 0 & 0 & 0 & 0 & 0 & 0 & 0 & 0 & 0 & 0 \\
\hline Mite & 0 & 0 & 1 & 0 & 0 & 0 & 0 & 0 & 0 & 0 & 0 & 0 & 0 & 0 & 1 \\
\hline \multicolumn{16}{|l|}{ Mollusca } \\
\hline Aplacophora & 0 & 0 & 0 & 1 & 0 & 0 & 0 & 0 & 0 & 0 & 0 & 0 & 0 & 0 & 0 \\
\hline Bivalvia & 0 & 0 & 0 & 0 & 0 & 3 & 10 & 0 & 0 & 1 & 0 & 1 & 0 & 0 & 1 \\
\hline Lucinidae & 0 & 0 & 0 & 5 & 0 & 0 & 0 & 0 & 0 & 0 & 0 & 0 & 0 & 0 & 0 \\
\hline Pectinidae & 0 & 0 & 0 & 1 & 0 & 0 & 0 & 0 & 0 & 0 & 0 & 0 & 0 & 0 & 0 \\
\hline \multicolumn{16}{|l|}{ Echinodermata } \\
\hline Ophiuroidea & 0 & 0 & 0 & 3 & 0 & 0 & 2 & 2 & 0 & 0 & 0 & 0 & 0 & 0 & 0 \\
\hline Nemertean & 0 & 0 & 0 & 0 & 0 & 0 & 1 & 0 & 0 & 0 & 0 & 0 & 0 & 0 & 0 \\
\hline Unknown Metazoan & 0 & 0 & 0 & 0 & 0 & 1 & 0 & 0 & 0 & 0 & 2 & 0 & 0 & 1 & 0 \\
\hline
\end{tabular}

declined with fairly constant low levels from $900-1050 \mathrm{~m}$ (ind $\mathrm{m}^{-2}$ ) (Fig. 3). Ambient metazoan macrofaunal densities at the colonization tray depths were $2405 \pm 506 \mathrm{ind}^{-2}$ at

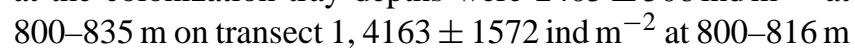
on transect 2 and $2035 \pm 0$ ind $\mathrm{m}^{-2}$ at $1147 \mathrm{~m}$ on transect 2 . General density trends with depth across the two transects were similar (Fig. 3).

\subsection{Colonizer and ambient composition}

Polychaetes were the dominant taxon in ambient sediments at all depths from 575 to $1150 \mathrm{~m}$ (Table 3). They comprised $100 \%$ of the fauna at $575 \mathrm{~m}$ and declined in proportion to only $50 \%$ at $800-900 \mathrm{~m}$, where Mollusca, Crustacea and Echinodermata became abundant (Fig. 4).
Polychaetes comprised the majority ( $93.8 \%$ ) of the 151 colonizers documented in this study (Table 4). The remaining colonizers were mollusks $(2.3 \%)$, crustaceans $(3.1 \%)$, echinoderms $(0.8 \%)$ and a single turbellarian and sipunculan (Table 3 ). Of the colonizing polychaetes, $85 \%$ were in the family Capitellidae; all of these were found at $1147 \mathrm{~m}$. This family of polychaetes was not collected from ambient sediments at $1147 \mathrm{~m}$, but was present at low densities $\left(103.3 \mathrm{ind} \mathrm{m}^{-2}\right)$ in ambient sediments at $802 \mathrm{~m}$ on transect 1 and at $930 \mathrm{~m}$ on transect 2 .

Calcareous foraminifera were present in trays at $542 \mathrm{~m}$. However, they were not quantified because they were too large to have been colonizers and, in most cases, it was not possible to determine whether they were alive or dead in sediments at the time of tray deployment. However, those 
Table 4. Macrofauna colonizing sediment trays deployed on the W. Indian margin.

\begin{tabular}{|c|c|c|c|c|c|c|c|c|c|c|c|c|c|c|c|c|}
\hline Date & $\begin{array}{l}11 \text { Oct } \\
2008\end{array}$ & $\begin{array}{l}11 \text { Oct } \\
2008\end{array}$ & $\begin{array}{c}11 \text { Oct } \\
2008\end{array}$ & $\begin{array}{l}11 \text { Oct } \\
2008\end{array}$ & $\begin{array}{c}12 \text { Oct } \\
2008\end{array}$ & $\begin{array}{l}12 \text { Oct } \\
2008\end{array}$ & $\begin{array}{l}12 \text { Oct } \\
2008\end{array}$ & $\begin{array}{c}12 \text { Oct } \\
2008\end{array}$ & $\begin{array}{l}2 \mathrm{Nov} \\
2008\end{array}$ & $\begin{array}{l}2 \mathrm{Nov} \\
2008\end{array}$ & $\begin{array}{l}2 \mathrm{Nov} \\
2008\end{array}$ & $\begin{array}{l}2 \mathrm{Nov} \\
2008\end{array}$ & $\begin{array}{l}1 \mathrm{Nov} \\
2008\end{array}$ & $\begin{array}{l}1 \mathrm{Nov} \\
2008\end{array}$ & $\begin{array}{l}1 \mathrm{Nov} \\
2008\end{array}$ & $\begin{array}{l}1 \mathrm{Nov} \\
2008\end{array}$ \\
\hline Depth (m) & 542 & 542 & 542 & 542 & 802 & 802 & 802 & 802 & 817 & 817 & 817 & 817 & 1147 & 1147 & 1147 & 1147 \\
\hline Replicate & A & B & $\mathrm{C}$ & $\mathrm{D}$ & A & $\mathrm{B}$ & $\mathrm{C}$ & $\mathrm{D}$ & A & B & $\mathrm{C}$ & $\mathrm{D}$ & $\mathrm{A}$ & B & $\mathrm{C}$ & $\mathrm{D}$ \\
\hline Fraction & $0-2$ & $0-2$ & $0-2$ & $0-2$ & $0-2$ & $0-2$ & $0-3$ & $0-2$ & $0-2$ & $0-2$ & $0-2$ & $0-2$ & $0-5$ & 0-3 & $0-5$ & $0-3$ \\
\hline Algal & ${ }^{13} \mathrm{C} /{ }^{15} \mathrm{~N}$ & ${ }^{13} \mathrm{C} /{ }^{15} \mathrm{~N}$ & No & No & ${ }^{13} \mathrm{C} /{ }^{15} \mathrm{~N}$ & ${ }^{13} \mathrm{C} /{ }^{15} \mathrm{~N}$ & No & No & ${ }^{13} \mathrm{C} /{ }^{15} \mathrm{~N}$ & ${ }^{13} \mathrm{C} /{ }^{15} \mathrm{~N}$ & No & No & ${ }^{13} \mathrm{C} /{ }^{15} \mathrm{~N}$ & ${ }^{13} \mathrm{C} /{ }^{15} \mathrm{~N}$ & No & No \\
\hline Treatment & Label & Label & algae & algae & Label & Label & algae & algae & Label & Label & algae & algae & Label & Label & algae & algae \\
\hline \multicolumn{17}{|l|}{ TAXON } \\
\hline \multicolumn{2}{|c|}{ Ampharetidae } & & & & & & & & & & & & & & & 2 \\
\hline \multicolumn{2}{|c|}{ Capitellidae } & & & & & & & & & & & & 9 & 48 & 36 & 11 \\
\hline \multicolumn{2}{|c|}{ Cirratulidae } & & & & & & 1 & 1 & & & & & & & & \\
\hline \multicolumn{2}{|c|}{ Cossuridae } & & & & & & 1 & & & & & & & & & \\
\hline \multicolumn{2}{|c|}{ Lumbrineridae } & & & & & & & & & & & 1 & & & & \\
\hline \multicolumn{2}{|c|}{ Sabellidae } & & & & & 1 & & & & & & & & & & \\
\hline \multicolumn{2}{|c|}{ Spionidae } & & & & & & & & & & & & 2 & 4 & & 1 \\
\hline \multirow{2}{*}{\multicolumn{2}{|c|}{$\begin{array}{l}\text { Prionospio sp. } \\
\text { Pygospio sp. }\end{array}$}} & & & & & & & 1 & & & & & & & & \\
\hline & & & & & & & & & & & & & & & & 1 \\
\hline \multicolumn{2}{|c|}{ Syllidae } & & & & & & & & & & & & & 1 & & \\
\hline \multicolumn{2}{|c|}{ Turbellarian } & & & & & & 1 & & & & & & & & & \\
\hline \multicolumn{2}{|l|}{ Sipunculan } & & & & & & 1 & & & & & & & & & \\
\hline \multicolumn{17}{|l|}{ Mollusca } \\
\hline \multirow{2}{*}{\multicolumn{2}{|c|}{ Aplacophora }} & & & & & & & & & & & & & & & \\
\hline & & & & & 1 & & & & & & & & & & & \\
\hline \multicolumn{2}{|c|}{ Gastropoda } & & & & & 1 & & 1 & & & & & & & & \\
\hline \multicolumn{17}{|l|}{ Crustacea } \\
\hline & & & & & & & & & & & & 1 & 1 & 1 & \\
\hline \multicolumn{2}{|c|}{ Cumacea } & & & & & & & & & & & & & 1 & & \\
\hline \multicolumn{2}{|c|}{ Echinodermata } & & & & & & & & & & & & & & & \\
\hline \multicolumn{2}{|c|}{ Ophiurodea } & & & & & & & & & & & & & 1 & & \\
\hline
\end{tabular}

individuals that had taken up labeled ${ }^{13} \mathrm{C}$ and ${ }^{15} \mathrm{~N}$ were presumably alive. No macrofauna were recovered in trays or sediments at $542 \mathrm{~m}$ (Table 3).

Colonizers at $802-817 \mathrm{~m}$ exhibited extremely low densities and diversities in this experiment. Of the 10 macrofaunal animals present in the $802 \mathrm{~m}$ colonization trays on transect 1 , five were polychaetes, one was a turbellarian, one a sipunculan and the other three were mollusks (Fig. 5b; Table 4). Two of the polychaetes in these four trays were cirratulids. The other three polychaetes at this site each represented a different family. Only a single polychaete specimen was present in the $817 \mathrm{~m}$ colonization trays on transect 2 (Table 4). Thus, in total, seven polychaete families, most represented by a single individual, were represented in the eight $800 \mathrm{~m}$ trays; none of these was found in sediment trays at $1147 \mathrm{~m}$ (Fig. 6). In ambient sediments at $800-835 \mathrm{~m}$, polychaetes $(55.6 \%)$, mollusks $(22.2 \%)$, crustaceans $(13.3 \%)$, and echinoderms $(8.9 \%)$ were all well represented (Fig. 5c).

Over $95 \%$ of the 120 individuals colonizing sediment trays at $1147 \mathrm{~m}$ were polychaetes. Although the proportion of polychaetes was higher than in the ambient sediments $(59 \%)$, densities were not significantly greater $(P=0.115)$ (Fig. 4). Of the colonizing polychaetes at this depth, Capitellidae accounted for $90.4 \%$ (Fig. 6). Also present were Spionidae (7\%), Ampharetidae (1.7\%), and Syllidae $(0.9 \%)$. Most of the polychaete families found in colonization trays were also present in ambient sediments at this depth;
Capitella was a notable exception. Ambient sediments at $1147 \mathrm{~m}$ contained $36.4 \%$ crustaceans, compared to only $3.33 \%$ crustaceans in colonization trays (Fig. 5).

Multidimensional scaling analysis of tray colonizers revealed a significant difference in community composition between colonizers at the sites with lower oxygen (802/817 m) and those where oxygen was higher (1147 $\mathrm{m}$ site). (ANOSIM $R=0.50 ; P=0.024$ ) (Fig. 7b); Because the same result is obtained with a presence/absence analysis (ANOSIM $R=$ $0.347 ; P=0.04$ ), we can attribute this different to composition rather than density effects. There was a significant difference in composition between colonization tray and ambient fauna (Fig. 7a), based on both faunal counts $(R=0.263$ : $P=0.002)$ and presence/absence $(R=0.148, P=0.038)$.

Species richness of tray colonizers was higher at $1147 \mathrm{~m}$ $(3.75 \pm 0.78 \mathrm{spp} . / \mathrm{tray})$ than at $802-817 \mathrm{~m}(1.38 \pm 0.55$ spp./tray) $\left(t_{10}=2.47 ; P=0.033\right)$ (Table 4$)$. Ambient cores $\left(54 \mathrm{~cm}^{2}\right)$, roughly $2 / 3$ the area of the colonization trays, exhibited average species richness per core of $0.0,8.25 \pm 1.25$, and $7 \pm 1.0$ at $535-542,800-817$, and $1147 \mathrm{~m}$, respectively (Table 3 ). Thus, the order of magnitude differences in oxygen concentration between 800 and $1147 \mathrm{~m}$ did not appear to affect local ambient richness, based on the limited number of cores examined. 


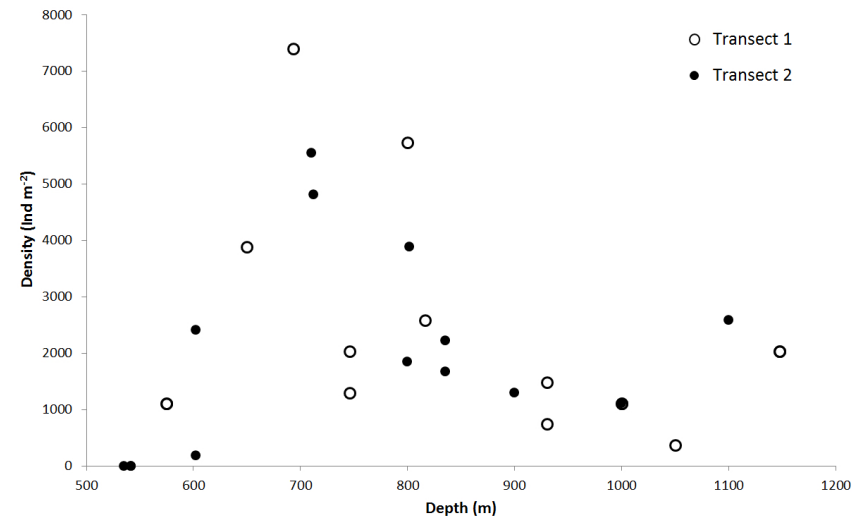

Fig. 3. Metazoan macrofaunal densities in ambient core samples $\left(54 \mathrm{~cm}^{2} \times 10 \mathrm{~cm}\right.$ deep) along two transects across the W. Indian margin.

\subsection{Phytodetritus effects}

The presence of ${ }^{13} \mathrm{C}$-labeled phytodetritus did not appear to influence the density, composition, or species richness of macrofaunal colonizers (Table 4, Fig. 8a, b; ANOSIM based on presence/absence $R=-0.06 P=0.698)$. At $802 \mathrm{~m}$ on transect 1 , faunal density in trays without labeled phytodetritus was double that of trays with labeled phytodetritus, however, at $817 \mathrm{~m}$ on transect 2 , the single colonizer entered a tray with no labeled phytodetritus. At $1147 \mathrm{~m}$ on transect 2, trays with labeled phytodetritus exhibited a density 1.3 times that of trays without phytodetritus (Fig. 8a). In all instances sample size ( $n=2$ per treatment at each depth) was too small to evaluate the significance of phytodetritus presence. Notably, Capitella sp. did not respond positively to the phytodetritus.

Two of the three animals found in trays with labeled phytodetritus at $802 \mathrm{~m}$ on transect 1 were mollusks and one was a polychaete. These numbers compare with four polychaetes, one turbellarian, one sipunculan and one gastropod in the two trays without added algae. At $1147 \mathrm{~m}$, polychaetes represented $94.1 \%(n=68)$ of the fauna in trays with labeled phytodetritus and $98.1 \%(n=52)$ in trays without labeled phytodetritus. Crustaceans comprised $4.4 \%$ and $1.9 \%$ of colonizers in trays with and without labeled phytodetritus, respectively (Table 4, Fig. 8b). Trays with phytodetritus collected fewer species than trays without phytodetritus at 802 $817 \mathrm{~m}$ ( 3 vs. 7 species) but had similar richness at $1147 \mathrm{~m} \mathrm{(6}$ vs. 5 species) (Table 4). There were no significant differences in species richness between trays with and without phytodetritus but sample sizes were small. Notably, cumaceans and ophiuroids appeared only at the deepest station, in trays with phytodetritus.

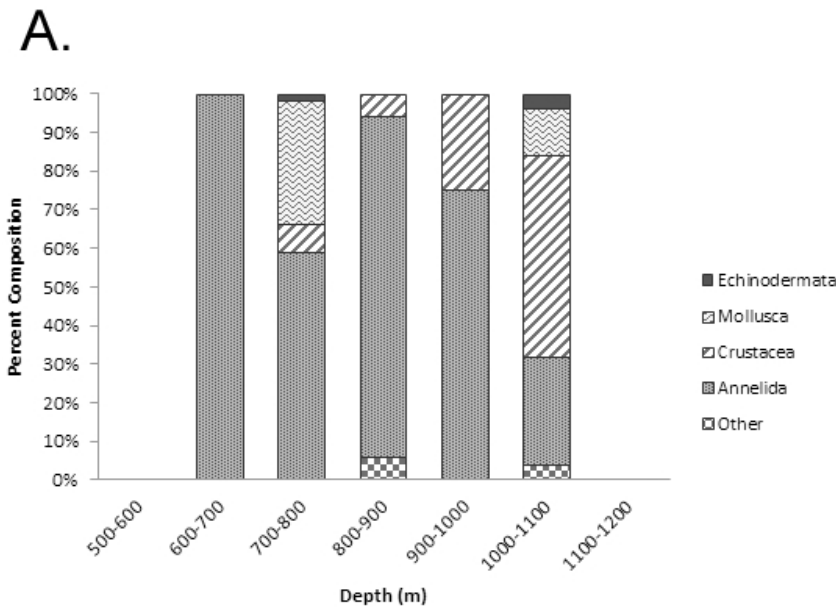

B.

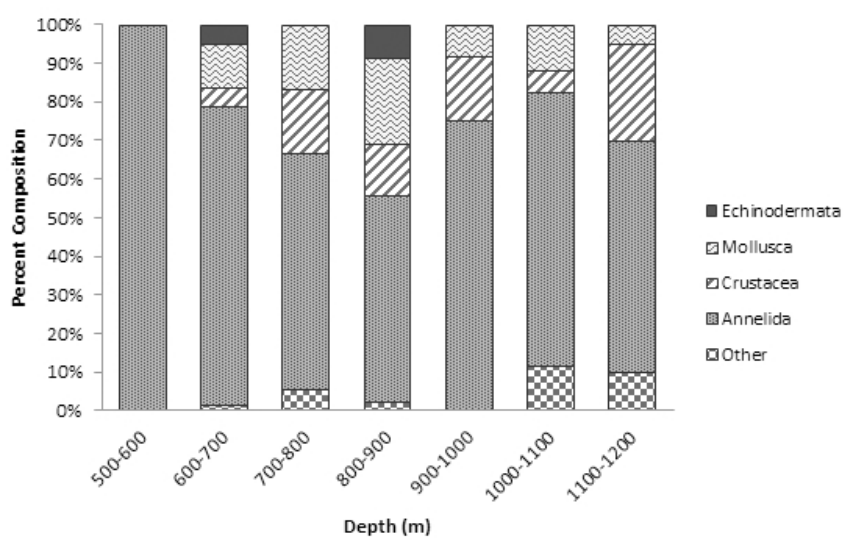

Fig. 4. Metazoan macrofaunal composition in ambient sediments sampled across the W. Indian margin along transect 1 (A) and transect 2 (B).

\subsection{Phytodetritus ingestion by colonizers}

The stable isotope signature of the phytodetritus added to colonization trays was $\delta^{13} \mathrm{C}=50626 \%$ and $\delta{ }^{15} \mathrm{~N}=57190 \%$. Although no metazoan macrofauna colonized trays with algae at $542 \mathrm{~m}$, several species of calcareous foraminifera, which were too large to have been colonizers, appeared to have consumed labeled phytodetritus in colonization trays at this depth (Table 5). Greatest uptake at $542 \mathrm{~m}$ was by Hoeglundina sp. $\left({ }^{13} \mathrm{C}=-11.6 \%\right.$, $\delta^{15} \mathrm{~N}=712 \%$; $\left.n=1\right)$ and Uvigerina sp. $\left({ }^{13} \mathrm{C}=-13.5 \%\right.$ o and $\delta^{15} \mathrm{~N}=368 ; n=3$ ), with little evidence of phytodetritus uptake by Lenticularia sp. $\left(\delta^{13} \mathrm{C}=-8.1 \%\right.$, $\delta^{15} \mathrm{~N}=-1.44 \%$; $n=2$ ) (Fig. 9a). However, Lenticularia sp. and several other taxa, including Hoeglundina sp., Chilostomella, and/or Globobulimina and an unidentified tubular form, appeared to have ingested labeled phytodetritus at $802 \mathrm{~m}$ (Table 5, Fig. 9a). No isotope data are available for the $817 \mathrm{~m}$ trays. 


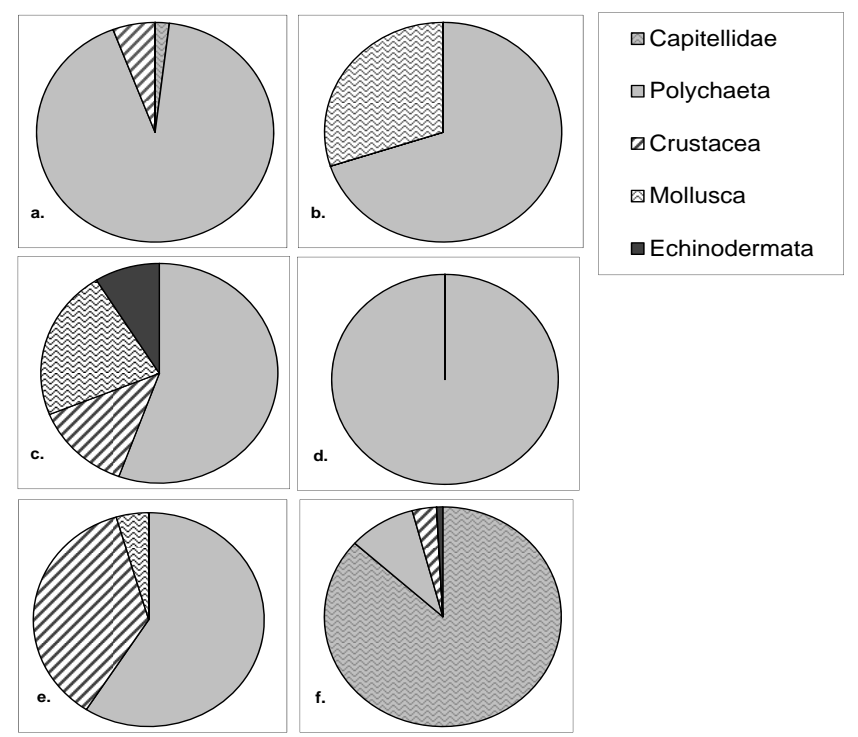

Fig. 5. Composition of higher macrofaunal taxa in ambient and colonization trays on the W. Indian margin. (a) Ambient fauna at 800 to $835 \mathrm{~m}$ on transect 1 ; (b) colonizers at $802 \mathrm{~m}$ on transect 1 ; (c) ambient fauna at 800 to $814 \mathrm{~m}$ on transect 2; (d) colonizers at $817 \mathrm{~m}$ on transect 2; (e) ambient fauna at $1148 \mathrm{~m}$ on transect 2; and (f) colonizers at $1147 \mathrm{~m}$ on transect 2 .

For metazoan macrofauna colonizing trays with labeled algae, the consumption of labeled phytodetritus and thus the departure from ambient isotopic signatures was greater at $1147 \mathrm{~m}$ than at the $802 \mathrm{~m}$ station (Table 5; Fig. 9B). The mean $(+1 \mathrm{SE})$ isotopic signatures of ambient macrofauna at $802 \mathrm{~m}$ was $\delta^{13} \mathrm{C}=-18.98+2.87 \%$ and $\delta^{15} \mathrm{~N}=7.1+4.1 \%$ o. In $802 \mathrm{~m}$ trays without phytodetritus, the mean isotopic signature of colonizers was $-23.4+0.9 \%$ o and $4.0+1.3 \%$ for $\delta^{13} \mathrm{C}$ and $\delta^{15} \mathrm{~N}$, respectively. In trays with phytodetritus, the respective macrofaunal signatures were $\delta^{13} \mathrm{C}=-21.0 \pm 4.2 \%$ and $\delta^{15} \mathrm{~N}=5.6 \pm 0.65 \%$. The $802 \mathrm{~m}$ trays with algae only had a gastropod and sabellid polychaete; these did not appear to take up any algae (Table 5). The one specimen colonizing at $817 \mathrm{~m}$ was not assayed for stable isotopes. Of the colonizers examined from $1147 \mathrm{~m}$ trays, $89 \%$ had $\delta^{15} \mathrm{~N}$ above ambient levels and $44 \%$ had $\delta^{13} \mathrm{C}$ above ambient levels (Table 5). Mean isotopic signatures of colonizers at $1147 \mathrm{~m}$ were $\delta^{13} \mathrm{C}=980.3 \pm 1845.5$ and $\delta^{15} \mathrm{~N}=1439.4 \pm 3382.7$ for trays containing labeled phytodetritus, and $\delta^{13} \mathrm{C}=-22.4 \pm 1.3$ and $\delta^{15} \mathrm{~N}=8.8 \pm 2.6$ for trays without phytodetritus. All of the colonizing capitellid polychaetes tested from trays with phytodetritus had $\delta^{13} \mathrm{C}$ signatures above ambient levels. The highest label uptake was by a capitellid $\left(\delta^{13} \mathrm{C}=5384.5 \%\right.$ ) and a cumacean $\left(\delta^{13} \mathrm{C}=2530.4 \%\right.$ ) . Two spionids and an amphipod did not take up labeled phytodetritus in trays at $1147 \mathrm{~m}$.

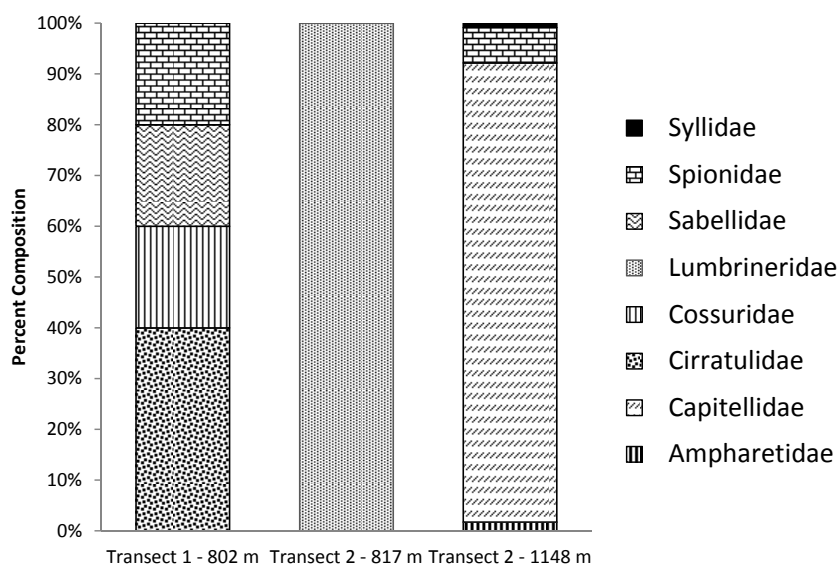

Fig. 6. Annelid family composition in colonization trays on the W. Indian margin. See Table 1 for tray deployment periods and locations.

\section{Discussion}

In this experiment we examined how colonizer density and composition differed at three water depths and oxygen levels, and explored the possible influence of phytodetritus on the type and abundance of colonizing invertebrates. We initially hypothesized that the abundance of organisms would increase with oxygen and depth, there would be a significant difference in community composition among stations, and that trays containing phytodetritus would support more colonizers. Our limited results suggest that oxygen exerts a strong effect on macrofaunal abundance and community composition, with core $\mathrm{OMZ}$ values of $0.7 \mu \mathrm{M} \mathrm{O}_{2}$ at $542 \mathrm{~m}$ (transect 1) inhibiting macrofaunal colonization completely. Colonizers were more abundant, with higher species richness at the better-oxygenated $1147 \mathrm{~m}$ station than at $802-817 \mathrm{~m}$. While the labeling experiments indicate that many colonizers are capable of consuming phytodetritus, the presence of the algae did not unidirectionally affect density or composition of tray colonizers.

Sample sizes in this study were very small and experimental duration was very short. These parameters were constrained by limited dive time and access to the sites. However, they provide a first glimpse into the dynamics of recolonization over very short periods in oxygen minimum zones.

\subsection{Density: ambient vs. colonizing fauna}

Density recovery rates in this study were fast at depths with higher oxygen, relative to other experiments with significantly longer duration. At $1147 \mathrm{~m}$ (this study), the density of animals in colonization trays on the Indian margin (3074 ind $\mathrm{m}^{-2}$ ) overshot ambient densities, reaching $150 \%$ of ambient densities after only 9 days. At methane seeps on the northern California margin $(525 \mathrm{~m})$, densities returned to only $50 \%\left(6827 \pm 753\right.$ ind $\left.^{-2}\right)$ of ambient levels after 
Table 5. Carbon and nitrogen stable isotopic signatures for colonizers of trays with and without labeled phytodetritus (algae) added. Background data are also given for sediments and protistans. Background data for macrofauna are given in Hunter et al. (2012) (Fig. 4).

\begin{tabular}{|c|c|c|c|c|c|c|}
\hline Depth (m) & Treatment & Major Taxon & Taxon ID & No. Individuals & $\delta^{13} \mathrm{C}(\mathrm{V}-\mathrm{PDB})$ & $\delta^{15} \mathrm{~N}(\mathrm{AIR})$ \\
\hline 542 & ${ }^{13} \mathrm{C} /{ }^{15} \mathrm{~N}$ Algae & Algae & Algae, labeled & N/A & 50625.6 & 57190.12 \\
\hline 542 & ${ }^{13} \mathrm{C} /{ }^{15} \mathrm{~N}$ Algae & Protista & Lenticularia sp. & 1 & -4.29 & 1.59 \\
\hline 542 & ${ }^{13} \mathrm{C} /{ }^{15} \mathrm{~N}$ Algae & Protista & Lenticularia sp. & 12 & -11.9 & -4.47 \\
\hline 542 & ${ }^{13} \mathrm{C} /{ }^{15} \mathrm{~N}$ Algae & Protista & Uvigerina sp. & 34 & -9.4 & 652.3 \\
\hline 542 & ${ }^{13} \mathrm{C} /{ }^{15} \mathrm{~N}$ Algae & Protista & Hoeglundina sp. & 23 & -11.6 & 711.86 \\
\hline 542 & ${ }^{13} \mathrm{C} /{ }^{15} \mathrm{~N}$ Algae & Protista & Uvigerina sp. & 40 & -12.74 & 415.76 \\
\hline 542 & ${ }^{13} \mathrm{C} /{ }^{15} \mathrm{~N}$ Algae & Protista & Uvigerina sp. & 30 & -18.31 & 36.02 \\
\hline 802 & No Algae & Polychaeta & Cirratulidae & 1 & -22.79 & 3.04 \\
\hline 802 & No Algae & Polychaeta & Cossuridae & 1 & -24.02 & 4.96 \\
\hline 802 & ${ }^{13} \mathrm{C} /{ }^{15} \mathrm{~N}$ Algae & Molluska & Gastropoda & 1 & -18.06 & 5.14 \\
\hline 802 & ${ }^{13} \mathrm{C} /{ }^{15} \mathrm{~N}$ Algae & Molluska & Sabellidae & 1 & -24.02 & 6.06 \\
\hline 802 & ${ }^{13} \mathrm{C} /{ }^{15} \mathrm{~N}$ Algae & Molluska & Bivalve & 1 & -3.49 & 12.85 \\
\hline 802 & ${ }^{13} \mathrm{C} /{ }^{15} \mathrm{~N}$ Algae & Ascidiacea & Ascidian & 1 & 235.3 & 2059.63 \\
\hline 802 & ${ }^{13} \mathrm{C} /{ }^{15} \mathrm{~N}$ Algae & Protista & Lenticularia sp. & 10 & 15.61 & 1712 \\
\hline 802 & ${ }^{13} \mathrm{C} /{ }^{15} \mathrm{~N}$ Algae & Protista & Hoeglundina sp. & 16 & -17.36 & 271.28 \\
\hline 802 & ${ }^{13} \mathrm{C} /{ }^{15} \mathrm{~N}$ Algae & Tube & Protozoa & & 16.9 & 131.44 \\
\hline 802 & ${ }^{13} \mathrm{C} /{ }^{15} \mathrm{~N}$ Algae & Protista & $\begin{array}{l}\text { Chilostomella sp. and, } \\
\text { Globobulimina sp. }\end{array}$ & 6 & -18.13 & 255.43 \\
\hline 802 & ${ }^{13} \mathrm{C} /{ }^{15} \mathrm{~N}$ Algae & Tube & Polychaete & 1 & -10.72 & 7.48 \\
\hline 1147 & No Algae & Polychaeta & Ampharetidae & 1 & -23.14 & 11.48 \\
\hline 1147 & No Algae & Polychaeta & Capitellidae & 2 & -24.48 & 3.09 \\
\hline 1147 & No Algae & Crustacea & Amphipoda & 1 & -22.62 & 8.45 \\
\hline 1147 & No Algae & Polychaeta & Spionidae & 1 & -24.32 & 8.98 \\
\hline 1147 & No Algae & Polychaeta & Pygospio & 1 & -22.47 & 7.88 \\
\hline 1147 & No Algae & Polychaeta & Capitellidae & 2 & -21.7 & 7.89 \\
\hline 1147 & No Algae & Polychaeta & Capitellidae & 3 & -20.55 & 12.2 \\
\hline 1147 & No Algae & Polychaeta & Capitellidae & 3 & -21.25 & 10.74 \\
\hline 1147 & No Algae & Polychaeta & Capitellidae & 3 & -21.8 & 7.62 \\
\hline 1147 & No Algae & Polychaeta & Capitellidae & 2 & -21.59 & 9.73 \\
\hline 1147 & ${ }^{13} \mathrm{C} /{ }^{15} \mathrm{~N}$ Algae & Polychaeta & Spionidae & 1 & -11.62 & 14.46 \\
\hline 1147 & ${ }^{13} \mathrm{C} /{ }^{15} \mathrm{~N}$ Algae & Polychaeta & Spionidae & 2 & -4.29 & 237.91 \\
\hline 1147 & ${ }^{13} \mathrm{C} /{ }^{15} \mathrm{~N}$ Algae & Polychaeta & Capitellidae & 1 & 348.58 & 167.19 \\
\hline 1147 & ${ }^{13} \mathrm{C} /{ }^{15} \mathrm{~N}$ Algae & Polychaeta & Capitellidae & 3 & 614.48 & 207.40 \\
\hline 1147 & ${ }^{13} \mathrm{C} /{ }^{15} \mathrm{~N}$ Algae & Polychaeta & Capitellidae & 1 & -22.21 & 111.37 \\
\hline 1147 & ${ }^{13} \mathrm{C} /{ }^{15} \mathrm{~N}$ Algae & Polychaeta & Capitellidae & 3 & 5384.46 & 10335.71 \\
\hline 1147 & ${ }^{13} \mathrm{C} /{ }^{15} \mathrm{~N}$ Algae & Polychaeta & Capitellidae & 2 & -15.14 & 67.34 \\
\hline 1147 & ${ }^{13} \mathrm{C} /{ }^{15} \mathrm{~N}$ Algae & Crustacea & Amphipoda & 1 & -2.04 & 20.56 \\
\hline 1147 & ${ }^{13} \mathrm{C} /{ }^{15} \mathrm{~N}$ Algae & Crustacea & Cumacea & 1 & 2530.38 & 1792.31 \\
\hline 835 & Background & Protista & $\begin{array}{l}\text { Globobulimina sp. and, } \\
\text { Chilostomella sp. }\end{array}$ & 15 & -9.34 & 5.46 \\
\hline 900 & Background & Protista & Reophax sp. & 3 & -21.71 & 3.02 \\
\hline 800 & Background & Protista & Allogromid & 1 & -20.21 & 3.72 \\
\hline 800 & Background & Protista & Nonionella sp. & 7 & -22.01 & 3.59 \\
\hline 540 & Background & & Sediment & & -20.38 & 5.53 \\
\hline 800 & Background & & Sediment & & -20.41 & 6.20 \\
\hline 814 & Background & & Sediment & & -20.22 & 6.02 \\
\hline 1145 & Background & & Sediment & & -20.59 & 7.49 \\
\hline
\end{tabular}

6 months in similar colonization trays (Levin et al., 2006). Short-term experiments deployed for 7 weeks on Fieberling Guyot in the eastern Pacific Ocean (585-635 m) had recov- ery rates of $6 \%\left(\sim 120 \mathrm{ind}^{-2}\right)$ and $31 \%\left(\sim 700\right.$ ind $\left.^{-2}\right)$ of ambient densities at two sites with low and high energy regimes (Levin and DiBacco, 1995). After 6 months, 
A.

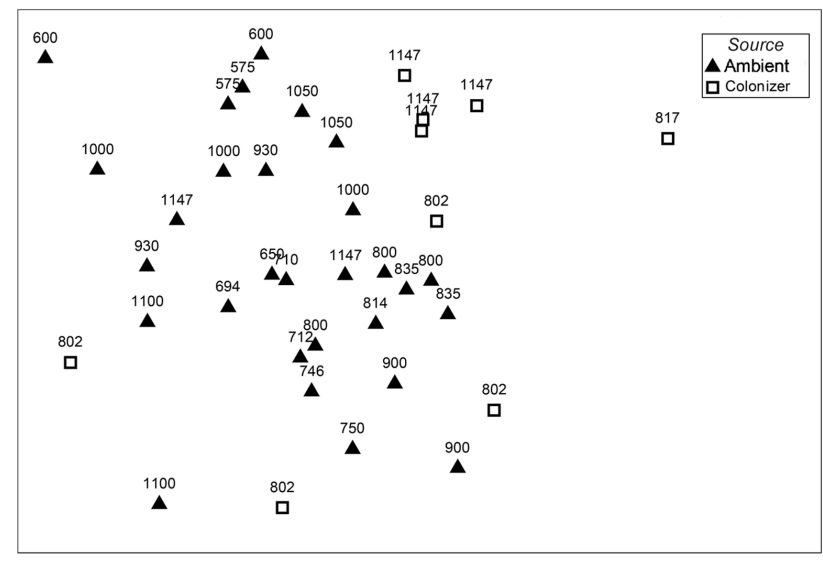

B.

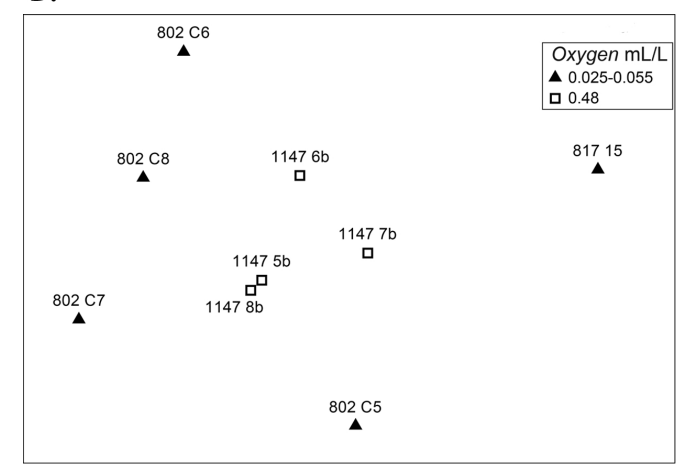

C.

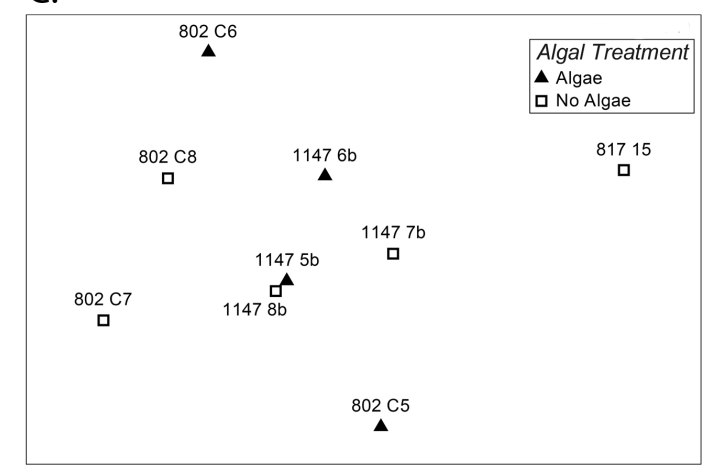

Fig. 7. Multidimensional scaling plot for metazoan macrofaunal community composition comparing (A) ambient fauna (closed symbols) and colonizers (open symbols), ANOSIM: $R=0.263, p=0.002$; (B) tray colonizers in high oxygen (open square) and low oxygen (closed triangle) stations, ANOSIM: $R=0.500, p=0.024$; and $(\mathbf{C})$ trays with labeled phytodetritus (closed symbols) and without phytodetritus (open symbols), ANOSIM: $R=-0.084, p=0.81$

recovery rates at these sites were $49 \%\left(\sim 700\right.$ ind $\left.^{-2}\right)$ and $75 \%\left(\sim 1250\right.$ ind $\left.^{-2}\right)$ of ambient densities (Levin and DiBacco, 1995).

However, other deep-sea studies have recorded density overshoots by colonizers. A 6 month study in the Bay of Biscay recorded colonizer densities 5 times higher than those in ambient sediment (Desbruyères et al., 1980, 1985). The ambient density in the Indian margin was notably lower than in the Bay of Biscay experiments. Other relatively short-term colonization experiments using a similar tray apparatus have yielded a variety of results. In a 23 day experiment in the Northwest Atlantic, macrofaunal densities in Thalassiosira

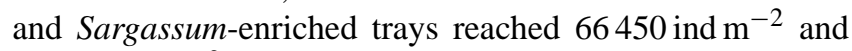
$16000 \mathrm{ind} \mathrm{m}^{-2}$, respectively, greatly exceeding ambient densities (Snelgrove et al., 1994, 1996). However, the unenriched controls did not exhibit the same ambient density overshoot seen in the enriched trays (Snelgrove, 1994, 1996). Macrofauna in the Indian margin exhibited a density overshoot at $1147 \mathrm{~m}$ but it was not correlated with the presence of phytodetritus and the overshoot was not as extreme as that observed in previous studies.

We observed slow recovery rates at the shallower, oxygendeprived depths of $802 \mathrm{~m}\left(1.2 \mu \mathrm{M} \mathrm{O}_{2}\right)$ and $817 \mathrm{~m}(2.2 \mu \mathrm{M}$ $\mathrm{O}_{2}$ ). Densities were $10.7 \%$ and $0.62 \%$ of ambient densities, respectively. Trays left at $802 \mathrm{~m}$ were deployed for only 4 days, a duration much shorter than in other studies. With only one exception (Snelgrove et al., 1994, 1996), no other published study has tested colonization trays in the deep sea for less than a month. This short duration is one factor that could explain the low colonizer density relative to the ambient conditions. Low oxygen is another factor that may explain this. Further studies are needed to understand the timescales at which oxygen interacts with other environmental attributes to influence assemblage resilience and recovery potential.

\subsection{Composition}

Colonization trays yielded a different assemblage of taxa compared to that found in ambient cores at corresponding 
A.

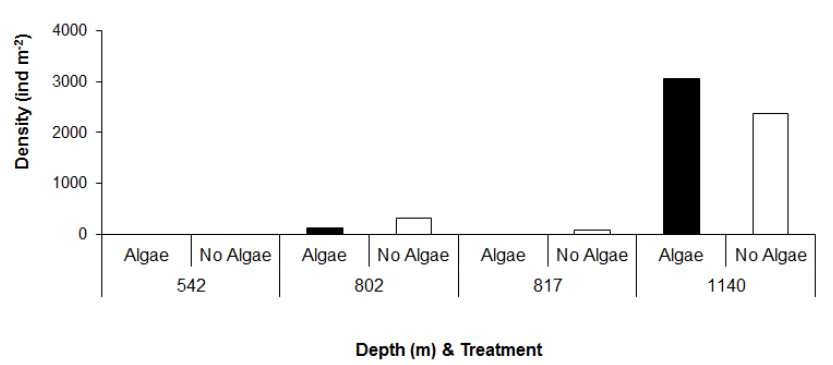

B.

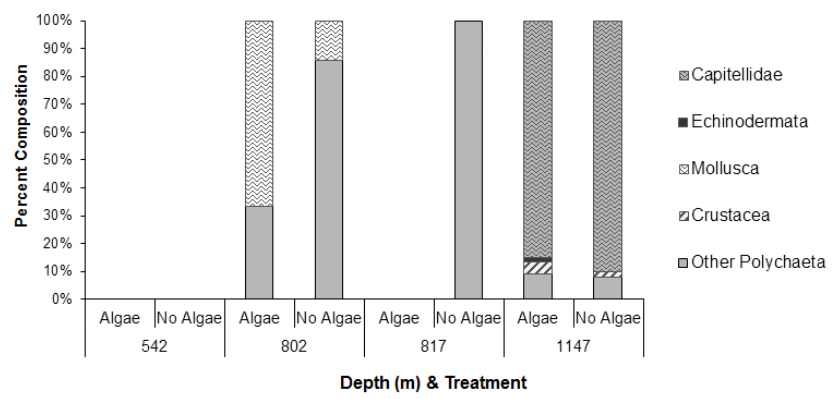

Fig. 8. (A) Comparison of average metazoan macrofaunal density in colonization trays with and without ${ }^{13} \mathrm{C} /{ }^{15} \mathrm{~N}$-labeled phytodetritus on the W. Indian margin. (B) Comparison of metazoan macrofaunal composition in colonization trays with and without phytodetritus on the W. Indian margin. Each bar reflects data combined for 2 trays. See Table 1 for tray deployment periods and locations.

depths. Colonization trays at $802 \mathrm{~m}$ on transect 1 contained 1 bivalve and 2 gastropods that accounted for $30 \%$ of the 10 animals colonizing. However, no mollusks were collected in ambient sediment at that depth, although sampling was limited. The proportion of polychaetes was $92 \%$ in ambient sediment and $70 \%$ (7 individuals) in colonization trays. This resembles the trends obtained by Levin at al. (2006) at a methane seep at the northern California margin, where polychaetes represented $57 \%$ of ambient and only $27 \%$ of colonization tray fauna. The ambient fauna obtained at $817 \mathrm{~m}$ on transect 2 was among the most diverse and abundant of any depth (Table 3). In contrast, within all 4 colonization trays only a single adult lumbrinerid was found. At $1147 \mathrm{~m}$, trays were strongly dominated by juvenile capitellid polychaetes. Other colonizers were of mixed size reflecting both adult and juvenile stages.

Capitellids alone reached an average density of 2,686 ind $^{-2}$ in $1147 \mathrm{~m}$ trays, whereas macrofauna in ambient sediments at that depth were dominated by syllid and paraonid polychaetes, amphipods, and tanaids, and contained no Capitellidae. Capitella is an opportunistic genus and will rapidly colonize disturbed sediments (Grassle and Grassle, 1976). Off the W. African margin, Capitellidae appeared at 1300 and $4000 \mathrm{~m}$ in enriched colonization trays, but were not exceptionally dominant (Menot et al.,
A.

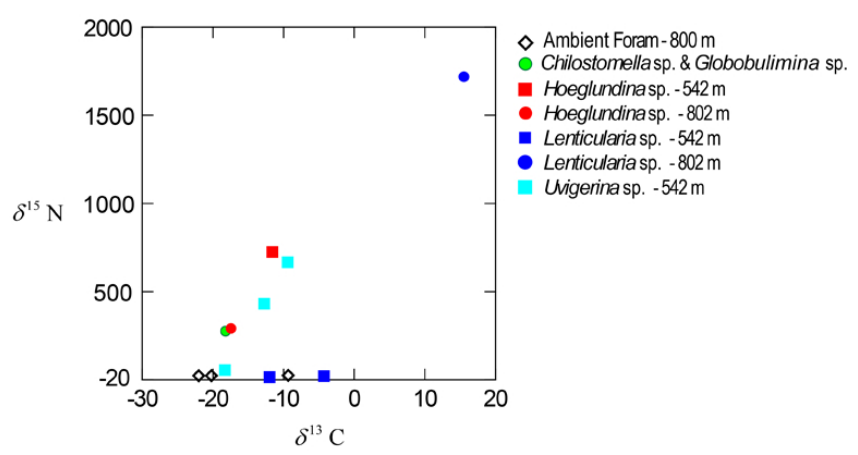

B.

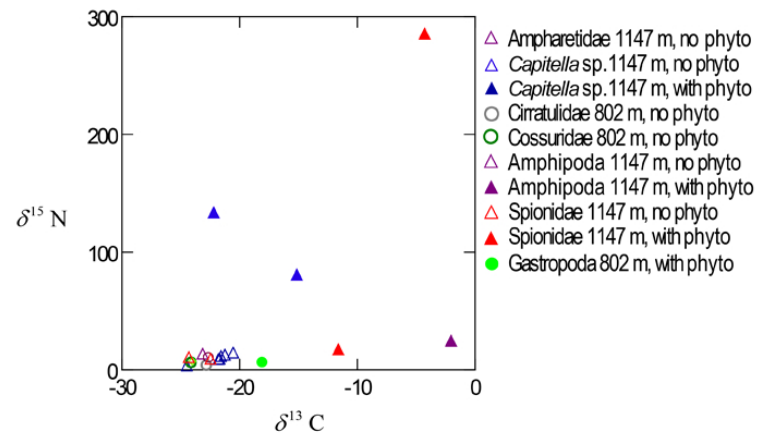

Fig. 9. (A) Dual isotope plot of foraminifera in colonization trays with ${ }^{13} \mathrm{C} /{ }^{15} \mathrm{~N}$ labeled phytodetritus added on transect 1 (Squares: $542 \mathrm{~m}$ trays; Circles: $800 \mathrm{~m}$ trays) and in ambient sediments (Diamond: $800 \mathrm{~m}$ ). (B) Dual isotope plot of metazoan macrofaunal colonizers in colonization trays with ${ }^{13} \mathrm{C} /{ }^{15} \mathrm{~N}$-labeled phytodetritus added (closed symbols $=$ with phyto) and those without (open symbols $=$ no phyto) from $802 \mathrm{~m}$ on transect 1 and $1147 \mathrm{~m}$ on transect 2 .

2009). In an experiment in the northwest Atlantic (900 m), capitellids accounted for half the animals in algae-enriched colonization trays after 23 days, but were absent in unenriched trays (Snelgrove et al., 1996). Capitellidae were also dominant in an experiment conducted south of New England (USA) at 1800 and $3600 \mathrm{~m}$. They were among the three most consistent colonizers and proved to be the most responsive to organic enrichment after 2 months (Grassle and Morse-Porteous, 1987). On the West Indian margin we did not observe the same discrepancy between trays with phytodetritus and without. Capitellidae represented $83.8 \%$ of the macrofauna in trays with algae and $90.4 \%$ in trays without (Fig. 8).

While most of the species in colonization trays did not match those found in ambient cores of the same depth, all of the polychaete taxa found in trays were present at some depths in Indian margin ambient sediments. These may have entered trays as planktonic larvae. It is also possible that we undersampled the ambient macrofaunal diversity, creating the appearance of a distinct disturbance colonizer fauna. The increasing incidence of large epifauna at greater depths 
and higher oxygen levels (Hunter et al., 2011) may have favored subsurface feeders like Capitella sp.

\subsection{Phytodetritus consumption}

Algae labeled with ${ }^{13} \mathrm{C}$ and ${ }^{15} \mathrm{~N}$ was added to colonization trays to determine which animals consume phytodetritus. The greatest uptake occurred at $1147 \mathrm{~m}$ where oxygen was highest; capitellid and spionid polychaetes and a cumacean ingested labeled phytodetritus, with $\delta^{13} \mathrm{C}$ values as high as $+5384.5 \%$ observed after 9 days (Fig. 9 b). But not all taxa took up the isotopic label; one spionid and an amphipod did not. In other studies, annelids exposed to labeled phytodetritus on the North Carolina (USA) slope at $850 \mathrm{~m}$ exhibited $\delta^{13} \mathrm{C}=-10$ to $+3,870 \%$, values significantly higher than that of ambient sediments $\left(\delta^{13} \mathrm{C}=-17.4\right.$ to $-23.5 \%$ ) (Blair et al., 1996), but non-annelid metazoans were slower to consume phytodetritus (Levin et al., 1999).

Our study, like others (Snelgrove et al., 1994, 1996), revealed a large response from cumaceans. In the NE Atlantic, Aberle and Witte (2003) found that the primary families to take up the labeled phytodetritus were Cirratulidae and Spionidae. The elevated role of these animals in the consumption of phytodetritus was attributed to their surface-deposit feeding lifestyle. The Capitellidae that were so abundant in our study are traditionally considered to be subsurface-deposit feeders. However, Levin et al. (2006) demonstrated that capitellid polychaetes can readily obtain carbon from labeled plant phytodetritus in shallow water. On the North Carolina (USA) slope ( $850 \mathrm{~m}$ ), only $25 \%$ of capitellids showed an increase in $\delta^{13} \mathrm{C}$ after 14 months of exposure to ${ }^{13} \mathrm{C}$ labeled phytodetritus, while $100 \%$ of cirratulids showed a response after the same amount of time (Levin et al., 1999). A similar result was also found in a Norwegian Fjord $(688 \mathrm{~m})$ where capitellid polychaetes showed little algal consumption after exposure to labeled algae for 2 to 14 days (Sweetman and Witte, 2008). While most of the capitellid individuals in our study exhibited an increased ${ }^{13} \mathrm{C} /{ }^{12} \mathrm{C}$ ratio, all Capitellidae had even more elevated ${ }^{15} \mathrm{~N} /{ }^{14} \mathrm{~N}$ ratios (Table 5). This suggests that ${ }^{15} \mathrm{~N}$ might be a more sensitive and reliable tracer for phytodetritus use than ${ }^{13} \mathrm{C}$; possibly it leaches from detritus into the dissolved organic matter pool and is quickly used by heterotrophic bacteria and then consumed by Capitella sp. Alternatively, C may be respired more readily than N, which could be sequestered within tissues. At $1147 \mathrm{~m}$, one of the spionids had an elevated ${ }^{15} \mathrm{~N} /{ }^{14} \mathrm{~N}$ ratio but the other was not significantly different than those in macrofauna from ambient sediments or unlabeled trays. A high degree of variation in mean isotopic signatures among taxa and even families is typical for phytodetritus labeling experiments (Levin et al., 1997, 1999; Aberle and Witte, 2003; this study).

During our cruise (YK08-11), comparable replicated isotope tracing experiments $(n=3)$ using the same labeled phytodetrital material were carried out directly on sediment in order to investigate rates and pathways of OM process- ing by the established macrofaunal community (Hunter et al., 2012). In these experiments, macrofauna was absent at $540 \mathrm{~m}$, and polychaetes were the most abundant taxon at the other three stations, with cirratulids and sabellids most abundant at $800 \mathrm{~m}$ on transect 1 , and oweniids and cirratulids most abundant at $800 \mathrm{~m}$ and $1100 \mathrm{~m}$ on transect 2 . In contrast to the colonizer community, $\mathrm{C}$ and $\mathrm{N}$ uptake by the established macrofauna community was dominated by cirratulids at both $800 \mathrm{~m}$ stations. At the $1100 \mathrm{~m}$ station, the majority of $\mathrm{C}$ and $\mathrm{N}$ uptake was spread more evenly among three polychaete families. In this study, only one individual of the genus Capitella was found in the mature community at $800 \mathrm{~m}$ on transect 1 (Hunter et al., 2012), suggesting that patterns of OM processing are likely to differ significantly during the transition from a pioneering to mature community.

Research by Woulds et al. $(2007,2009)$ on the Pakistan margin has shown significant effects of oxygen on the taxa responsible for phytodetritus processing. Protists (foraminifera) dominate phytodetritus consumption at oxygen concentrations below $5 \mu \mathrm{M}$ whereas metazoan macrofauna dominate at higher oxygen levels. Protists were not quantified in our experiments, but they were present in trays at $542 \mathrm{~m}$ and $802 \mathrm{~m}$ and clearly took up labeled $\mathrm{N}$ and $\mathrm{C}$, whereas metazoan phytodetritus uptake was significant only at higher oxygen levels (Fig. 9). Where the foraminifera in the recolonization trays originated from is unclear. They are relatively large calcareous species that were retained on a $300 \mu \mathrm{m}$ mesh sieve. Macrofauna-sized agglutinated foraminifera were reported from colonization trays by Kaminski et al. (1988), but these experiments were conducted over a 9-month time period. It would be impossible for individuals of this size to develop from colonizing propagules or juveniles within a period of days. There are several other possible explanations: the foraminifera may have crawled into the trays across the flat collar, been resuspended and wafted into the trays during submersible operations, or they survived freezing at $-20^{\circ} \mathrm{C}$ and sonication. Whatever their origin, the fact that they took up the label from phytodetritus demonstrates that they were alive during the experiment.

\subsection{Effects of phytodetritus on density and composition}

We observed no significant effect of phytodetritus additions on the density or composition of macrofaunal colonizers, which were similar in trays with and without phytodetritus additions. In earlier enrichment studies in the northwest Atlantic (Snelgrove et al., 1992, 1994, 1996) and off southern California (Levin and Smith, 1984), unenriched trays never attained ambient densities and enriched trays greatly exceeded ambient densities. Opportunistic colonizers have been shown to respond more rapidly in situations with organic enrichment (Smith and Hessler, 1987). In a study of colonization response to organic enrichment on the W. African margin, the results of Menot et al. (2009) suggested 
that communities under natural disturbances would exhibit high colonization rates driven by opportunistic species able to maintain populations in patchily distributed, disturbed areas. In our experiments, additions were designed to detect phytodetritus consumption rather than enhance organic matter availability. Thus, the addition of phytodetritus represented a $<1 \%$ enrichment of carbon in the surface $1 \mathrm{~cm}$ of sediment. Nevertheless, similar organic matter additions have prompted benthic community responses in previous studies (e.g., Menot et al., 2009). This may have been partly due to the freshness or high "food quality" of added organic matter compared to that which normally arrives at the deepsea floor (e.g., Woulds et al., 2007). The lack of response to phytodetritus observed in our colonization trays is consistent with findings at an oligotrophic setting at $4000 \mathrm{~m}$ on the W. African margin, where macrofaunal densities were not positively correlated with organic enrichment, but different from those at $1300 \mathrm{~m}$ where 0.3 and $1 \%$ enrichment yielded enhanced macrofaunal densities (Menot et al., 2009).

\subsection{Factors influencing colonization}

As shown by Grassle and Morse-Porteous (1987), experiment deployment time may be critical in determining the density and composition of the colonizers. Colonizers that respond rapidly to organic enrichment may be present after 2 months but could get out-competed after 10 months. The time range of 4 to 9 days does not reveal the complete successional response.

Recolonization of natural sediment has been shown to occur more readily than in trays with prefrozen sediment and also to attract an assemblage of macrofauna more similar to ambient assemblages (Smith, 1985). The heavy colonization of the $1147 \mathrm{~m}$ trays by Capitella is consistent with past observations of Capitella as a disturbance opportunist (Grassle, Morse-Porteous, 1987; Snelgrove et al., 1994, 1996) that is rare in undisturbed ambient sediments in deep water. In a 6 month experiment, colonization of trays containing coastal sediment was $1 / 3$ that of trays with sediment from the abyssal depths where the experiment took place, despite higher OM content in the coastal sediment (Desbruyères et al., 1980). Colonization trays have been posited to cause altered hydrodynamics and isolation, as well as having an arbitrary size (Smith, 1985). Although the trays used here are hydrodynamically unbiased and scour was limited, the tray design may exclude species that "crawl" within a limited area and preferentially select those settling or advected from the water column. This may contribute to differences between ambient fauna and colonizers.

\section{Conclusions}

This study was the first to examine the effects of reduced oxygen concentration on continental slope early colonization and to draw comparisons to ambient density and composition. Few colonization experiments have been conducted in the Indian Ocean; most experiments of this type have been conducted in the Pacific and Atlantic oceans. This study was also unusual in that deployment times were shorter than in any other reported deep-water colonization experiment. Results indicate the potential for rapid colonization by opportunists if oxygen is sufficient, but little colonization when it is not. As little as 9 days is enough time to overshoot ambient density by $150 \%$ at oxygen concentrations of around $0.5 \mathrm{mLL}^{-1}(22 \mu \mathrm{M})$. Most of the colonizing taxa were present in ambient sediments.

The type of information generated in this study is relevant to understanding consequences for benthic ecosystems exposed to or recovering from declining oxygen levels. Increased extent of both anthropogenic dead zones (Diaz and Rosenberg, 2008) and natural oxygen minimum zones (Stramma et al., 2008; 2010) are exposing more shelf and slope communities to hypoxic conditions (Bograd et al., 2008; Chan et al., 2008). Colonization by benthos will occur following hypoxia-induced mortality on shelves.

Understanding of colonization dynamics and ensuing succession is also important for management of areas subject to direct human disturbance. Trawling, oil spills, or seabed mining can all create scenarios in OMZs where colonization occurs following disturbance. Phosphate mining is being considered within several OMZs globally. We note that the small scales on which we studied colonization are not likely to paint a true picture of colonization dynamics following a large-scale mining or trawling disturbance. Thus, despite the fact that we saw rapid colonization at $1150 \mathrm{~m}$ off $\mathrm{W}$. India within a week, this cannot be used as evidence for rapid colonization of seafloor habitats. Additional research is needed to address subsequent changes in colonizer assemblages over longer time periods and larger spatial scales. Further exploration of colonization trends across hydrographic gradients and their consequences for ecosystem services is warranted.

Acknowledgements. We thank the captain and crew of the RV Yokosuka and the pilots and staff of the Shinkai 6500 for their assistance with the field operations. We thank the scientists participating in cruise number YK08-11 for their assistance at sea and in making auxiliary measurements, especially Kazumasa Oguri, Will Hunter, and Hidetaka Nomaki. We thank Ray Lee for assistance with stable isotope analyses and we thank L. Menot and two anonymous reviewers for their insightful comments on an earlier draft of the manuscript. This research was supported by JAMSTEC and grant no. ERI 008427 from The Carnegie Trust (to UW).

Edited by: S. W. A. Naqvi

\section{References}

Aberle, N. and Witte, U.: Deep-sea macrofauna exposed to a simulated sedimentation event in the abyssal NE Atlantic: in situ 
pulse-chase experiments using ${ }^{13} \mathrm{C}$-labelled phytodetritus, Mar. Ecol. Prog. Ser., 251, 37-47, 2003.

Blair, N. E., Levin, L. A., DeMaster, D. J., and Plaia, G.: The shortterm fate of fresh algal carbon in continental slope sediments. Limnol. Oceanogr., 41, 1208-1219, 1996.

Bograd, S. J., Castro, C. G., Di Lorenzo, E., Palacios, D. M., Bailey, H., Gilly, W., and Chavez, F. P.: Oxygen declines and the shoaling of the hypoxic boundary in the California Current, Geophys. Res. Lett., 35, L12607. doi:10.1029/2008GL034185, 2008.

Buhring, S. I., Lampadariou, N.: Moodley, L., Tselepides, A., and Witte, U., Benthic microbial and whole-community responses to different amounts of ${ }^{13} \mathrm{C}$-enriched algae: in situ experiments in the deep Cretan Sea (Eastern Mediterranean), Limnol. Oceanogr., 51, 157-165, 2006.

Chan, F., Barth, J. A., Lubchenco, J., Kirincich, A., Weeks, H., Peterson, W. T., and Menge, B. A.: Emergence of anoxia in the California Current large marine ecosystem, Science, 319, p. 920, 2008

Desbruyeres, D., Bevas, J., and Khripounoff, A.: Un cas de colonization rapide d'une sediment profound, Oceanol. Acta, 3, 285291, 1980.

Desbruyeres, D., Deming J.W., Dinet, A., and Khripounoff, A.: Reactions de l'ecosysteme benthique profound aux perturbations: noveaux resultants experimenteaux, in: Peuplements profonds du Golfe de Gascogne, edited by: Laubier, L. and Monniot, C., Institute Franceais de Recherche pour l'Exploration de la Mer, 193209, 1985.

Díaz, R. J. and Rosenberg, R.: Spreading dead zones and consequences for marine ecosystems, Science, 321, 926-929, 2008.

Gooday, A. J., Bett, B. J., Escobar, E., Ingole, B., Levin, L. A., Neira, C., Raman, A. V., and Sellanes, J.: Habitat heterogeneity and its relationship to biodiversity in oxygen minimum zones, Mar. Ecol., 31, 125-147, 2010.

Grassle, J. P. and Grassle, J. F.: Sibling species in the marine pollution indicator Capitella (Polychaeta), Science, 19, 567-569, 1976.

Grassle, J. F. and Morse-Porteous, L. S.: Macrofaunal colonization of disturbed deep-sea environments and the structure of deep-sea communities, Deep Sea Res., 34, 1911-1950, 1987.

Helly, J. and Levin, L. A.: Global distribution of naturally occurring marine hypoxia on continental margins, Deep Sea Res., 51, 11591168, 2004.

Hughes, D. J., Lamont, P. A., Levin, L. A., Packer, M. and Gage, J. D.: Macrofaunal community structure and bioturbation across the Pakistan margin oxygen minimum zone, north-east Arabian Sea, Deep Sea Res. II., 56, 434-448, 2010.

Hunter, W. R., Levin, L. A., Kitazato, H., and Witte, U.: Macrobenthic assemblage structure and organismal stoichiometry control faunal processing of particulate organic carbon and nitrogen in oxygen minimum zone sediments, Biogeosciences, 9, 993-1006, doi:10.5194/bg-9-993-2012, 2012.

Ingole, B. S., Sautya, S., Sivadas, S., Singh, R., and Nanajkar, M.: Macrofaunal community structure in the western Indian continental margin including the oxygen minimum zone, Mar. Ecol., 31, 148-166, doi:10.1111/j.1439-0485.2009.00356.x, 2010.

Kaminski, M. A., Grassle, J. F., and Whitlach, R. B.: Life history and recolonization among agglutinated foraminifera in the Panama Basin, in: Second Workshop on Agglutinated Foraminifera, edited by: Gradstein, M. F. and Rögl, F., Abhand- lungen der Geologischen Bundesanstalt, Wien, 41, 229-243, 1988.

Keeling, R. F., Kórtzinger, A., and Gruber, N.: Ocean deoxygenation in a warming world, Annu. Rev. Mar. Sci., 2, 199-229, 2010.

Levin, L. A.: Oxygen minimum zone benthos: adaptation and community response to hypoxia, Oceanogr. Mar. Biol. Ann. Rev., 41, $1-45,2003$.

Levin, L. A., Blair, N., DeMaster, D. J., Plaia, G., Fornes, W., Martin, C. and Thomas, C.: Rapid subduction of organic matter by maldanid polychaetes on the North Carolina slope, J. Mar. Res., 55, 595-611, 1997.

Levin, L. A, Blair, N., Martin, C., DeMaster, D., Plaia, G., and Thomas, C.: Macrofaunal processing of phytodetritus at two sites on the Carolina margin: In situ experiments using ${ }^{13} \mathrm{C}$ labeled diatoms, Mar. Ecol. Progr. Ser., 182, 37-54, 1999.

Levin, L. A. and DiBacco, C.: The influence of sediment transport on short-term recolonization by seamount infauna, Mar. Ecol. Progr. Ser., 123, 163-175, 1995.

Levin, L. A. and Gage, J. D.: Relationships between oxygen, organic matter and the diversity of bathyal macrofauna, Deep Sea Res., 45, 129-163, 1998.

Levin, L. A. and Smith, C. R.: Response of fauna to disturbance and enrichment in the deep sea: a sediment tray experiment, Deep Sea Res., 31, 1277-1285, 1984.

Levin L. A., Gage J. D., Martin C., and Lamont P. A.: Macrobenthic community structure within and beneath the oxygen minimum zone, NW Arabian Sea, Deep Sea Res. II, 47, 189-226, 2000.

Levin, L. A., Ziebis, W., Mendoza, G. F., Growey-Cannon, V., and Walther, S.: Recruitment response of methane-seep macrofauna to sulfide-rich sediments: An in situ experiment, J. Exp.. Mar. Biol. Ecol., 330, 132-150, 2006.

Levin L. A., Whitcraft, C., Mendoza, G. F., Gonzalez, J. P., and Cowie, G.: Oxygen and organic matter thresholds for benthic faunal activity: a case study across the Pakistan margin oxygen minimum zone (700-1100 m), Deep Sea Res. II, 56, 449-471, 2009.

Levin, L. A., Ziebis, W., Mendoza, G. F., Bertics, V. J., Washington, T., Gonzalez, J., Thurber, A. R., Ebbe, B., and Lee, R. W.: Ecological release and niche partitioning under stress: Lessons from dorvilleid polychaetes in sulfidic sediments at methane seeps, Deep Sea Res. II, 92, 214-233, 2013.

McClatchie, S., Goericke, R., Cosgrove, R., Auad, G., \& Vetter, R. Oxygen in the Southern California Bight: Multidecadal trends and implications for demersal fisheries, Geophys. Res. Lett., 37, L19602. doi:10.1029/2010GL044497, 2010.

Menot, L., Crassous, P., Desbruyeres, D., Galeron, J., Khripounoff, A., and Sibuet, M.: Colonization patterns along the equatorial West African margin: Implications for functioning and diversity maintenance of bathyal and abyssal communities, Deep Sea Res. II, 56, 2313-2325, 2009.

Pacheco, A. S., Laudien, J., Thiel, M., Oliva, M., and Heilmayer, O.: Succession and seasonal onset of colonization in subtidal hardbottom communities off northern Chile, Mar. Ecol., 32, 75-87, doi:10.1111/j.1439-0485.2010.00398.x, 2011.

Pozzato, L., van Oevelen, D., Moodley, L., Soetaert, K., and Middelburg, J. J.: Carbon processing at the deep-sea floor of the Arabian Sea oxygen minimum zone: A tracer approach, J. Sea Res., 78, 45-58, 2013. 
Rabalais, N. N., Díaz, R. J., Levin, L. A., Turner, R. E., Gilbert, D., and Zhang, J.: Dynamics and distribution of natural and humancaused hypoxia, Biogeosciences, 7, 585-619, doi:10.5194/bg-7585-2010, 2010.

Ramirez-Llodra, E., Tyler, P. A., Baker, M. C., Bergstad, O. A., Clark, M. R., Escobar, E., Levin, L. A., Menot, L., Rowden A. A., Smith, C. R., and Van Dover, C. L.: Man and the last great wilderness: Human impact on the deep sea, PLoS ONE, 6, e22588, doi:10.1371/journal.pone.0022588, 2011

Smith, C. R.: Colonization studies in the deep sea: are results biased by experimental designs?, in: Nineteenth European Marine Biology Symposium, edited by: Gibbs, P. E., Cambridge University Press, Cambridge, 183-190, 1985.

Smith, C. R. and Hessler, R. R.: Colonization and succession in deep-sea ecosystems, Trends Ecol. Evol., 2, 359-363, 1987.

Snelgrove, P. V. R. and Smith, C. R.: A riot of species in an environmental calm: the paradox of the species-rich deep sea, Oceanogr. Mar. Biol. Ann. Rev., 40, 311-342, 2002.

Snelgrove, P. V. R., Grassle, J. F., and Petrecca, R. F.: The role of food patches in maintaining high deep-sea diversity: field experiments using hydrodynamically unbiased colonization trays, Limnol. Oceanogr., 37, 1543-1550, 1992.

Snelgrove, P. V. R., Grassle, J. F., and Petrecca, R. F.: Macrofaunal response to artificial enrichments and depressions in a deep-sea habitat, J. Mar. Res., 52, 345-369, 1994.

Snelgrove, P. V. R., Butman, C. A., and Grassle, J. F.: Potential flow artifacts associated with benthic experimental gear: deepsea mudbox examples, J. Mar. Res., 53, 821-845, 1995.
Snelgrove, P. V. R., Grassle, J. F., and Petrecca, R. F.: Experimental evidence for aging of food patches as a factor contributing to high deep-sea macrofaunal diversity, Limnol. Oceanogr., 41, 605-614, 1996.

Stramma, L., Johnson, G. C., Sprintall, J., and Mohrholz, V.: Expanding oxygen-minimum zones in the tropical oceans, Science, 320, 655-658, 2008.

Stramma, L., Schmidt, S., Levin, L. A., and Johnson, G. C.: Ocean oxygen minima expansions and their biological impacts, Deep Sea Res., 210, 587-595, 2010.

Sweetman A. and Witte U.: Macrofaunal response to phytodetritus in a bathyal Norwegian fjord, Deep Sea Res., 155, 1503-1514, 2008.

Witte, U., Aberle, N., Sand, M., and Wenzhofer, F.: Rapid response of deep-sea benthic community to POM enrichment: an in situ experimental study, Mar. Ecol. Prog. Ser., 51, 27-36, 2003.

Woulds, C., Cowie, G. L., Levin, L. A., Andersson, J. H., Middelburg, J. J., Vandewiele, S., Lamont, P. A., Larkin, K. E., Gooday, A. J., Schumacher, S., Whitcraft, C., Jeffreys, R., and Schwartz, M.: Oxygen as a control on sea floor biological communities and their roles in sedimentary carbon cycling, Limnol. Oceanogr., 52, 1698-1709, 2007.

Woulds, C., Cowie, G., Andersson, J. H., Middelburg, J. J., and Levin, L. A.: ${ }^{13} \mathrm{C}$ tracer studies on the short-term fate of organic carbon in marine sediments: comparing the Pakistan margin to other regions, Deep Sea Res. II, 56, 393-402, 2009. 